Yunjong Eo*

\title{
Structural changes in inflation dynamics: multiple breaks at different dates for different parameters
}

\author{
DOI 10.1515/snde-2015-0041
}

\begin{abstract}
I consider complicated patterns of structural breaks in postwar quarterly US inflation rates based on the CPI and the GDP deflator over the period from 1953:Q1 to 2013:Q4. Bayesian model selection procedures suggest that the two inflation measures had distinct structural changes in different parameters as well as at different dates. CPI inflation experienced a dramatic drop in persistence around the early 1980s, but GDP deflator inflation remains persistent throughout the postwar sample period. The residual variance for both inflation measures switched from a low volatility regime to a high volatility regime in the early 1970s, but returned to another low volatility regime at different dates: the early 1980s for GDP deflator inflation and the early 1990s for CPI inflation. The residual variance for CPI inflation has increased again since the early 2000s, while GDP deflator inflation has remained less volatile. I do not find evidence of a structural shift in the unconditional mean of either measure of inflation. When reviewing the recent literature, considerable controversy exists over the structural break in inflation persistence around the early 1980s but this appears to be dependent on the measures of inflation, as highlighted by the empirical findings in this paper.
\end{abstract}

Keywords: Bayesian analysis; inflation dynamics; multiple-group changepoint; persistence; structural breaks; UC-SV model.

JEL classification: C11; C22; E31.

\section{Introduction}

In this paper, I make inferences about complicated patterns of structural breaks in inflation dynamics. An autoregressive (AR) model is used for studying structural shifts in three parameter groups: the unconditional mean, a group of autoregressive coefficients, and the residual variance. The choice of parameter groups are potentially related to structural interpretations of changes in inflation dynamics. Some parameters could have a different number of structural breaks and/or at different dates from others. Also, structural breaks are modeled as abrupt changes to identify potential regime shifts in economic structure such as a long-run inflation target, monetary policy, and price-setting behavior.

Changes in the group of AR coefficients, related to inflation persistence, can be understood through shifts in structural parameters in the New Keynesian Phillips curve. Shifts in inflation persistence can be

1 Fuhrer (2010) examines whether various measures of US inflation have a unit root for several sets of sample periods using the ADF test and the Phillips-Perron test. He finds that the results are ambiguous but one may safely assume that inflation does not contain an important unit root for the period since the mid-1980s. Fuhrer (2010) claims that if the central bank has a stable inflation goal, inflation would not have a unit root and this can be the case of the US economy. When taking his argument into account, I focus on the possibility of abrupt changes in trend inflation as in Levin and Piger (2006) rather than stochastic trend which evolves as a driftless random walk as in Cogley, Primiceri and Sargent (2010).

*Corresponding author: Yunjong Eo, School of Economics, University of Sydney, Sydney 2006, Australia, Phone: +612 9351 3075, Fax: +612 9351 4341, e-mail: yunjong.eo@sydney.edu.au 
caused by changes in firms' price setting behavior, marginal cost dynamics, or monetary policy. ${ }^{2}$ Shifts in inflation persistence have crucial implications for policymakers. For example, Solow (1968) and Tobin (1968) consider a test for the natural rate hypothesis using a simple Phillips curve with unemployment rate. When inflation is considered to be less persistent, the natural rate hypothesis is likely to be rejected. In such a case, policymakers may feel a strong temptation to exploit an illusory inflation-unemployment trade-off, resulting in high inflation as in the 1970s. ${ }^{3}$

The unconditional mean in AR models is associated with trend inflation, which is potentially set by the cental bank. Thus, one can make inferences about shifts in the central bank's long-run inflation target when studying structural breaks in the unconditional mean. However, the changes in trend inflation would not be identified if we consider breaks in the intercept because the shifts in the intercept may provide mixed information on breaks in the unconditional mean and persistence.

In addition, Stock and Watson (2003) and Kim, Nelson and Piger (2004) suggest the possibility of structural changes in the volatility of inflation as potentially related to real activity and supply shocks. This shift can be captured when taking into account the change in the residual variance.

In short, these complex patterns of structural breaks in different parameters reflect the possibility that the parameter changes are due to the shifts in structural parameters of macroeconomic models. ${ }^{4}$ To understand the structural dynamics of inflation clearly, it is essential above all to investigate whether there have been breaks. If so, which parameters are subject to these shifts? Do they undergo these changes at the same time? To answer these questions, it is important to identify the parameters subject to changes at a particular time and distinguish them from unchanged parameters.

I take a Bayesian approach to estimate and compare the complex structural break models, which are potentially non-nested. However, in the literature all the parameters subject to the changes are assumed to have the structural shifts at the same date either in pure or partial structural change models. ${ }^{5}$ For example, Chib (1998) considers single-group changepoint models by interpreting structural changes for all the parameters as regime transitions that follow a first-order Markov processes. Wang and Zivot (2000) treat structural break dates as additional parameters and sample break dates from conditional distributions of break dates with flat priors.

Thus, these approaches in the literature are not suitable for making inferences about the complicated patterns of structural changes considered in this paper. To overcome the limitation I modify Chib's (1998) approach, augmenting each parameter (group) with its corresponding independent regime indicator variable. Note that it is possible to sort several parameters into a group and make the group of parameters undergo structural changes at the same dates, as in the approach for persistence parameters adopted in this paper. The modified approach allows multiple parameters (groups) to undergo mutually independent structural breaks at different dates with the different number of breaks. This multiple-group changepoint model can explore all the possible patterns of structural breaks efficiently. The number of structural breaks and the form

2 For the effect of monetary policy regime on persistence in inflation, see the discussion in Davig and Doh (2014) and references therein.

3 For a more detailed discussion, see Cogley and Sargent (2002), Pivetta and Reis (2007), and references therein. 4 For example, consider a simple New Keynesian Phillips curve (NKPC) which has the form

$$
\hat{\pi}_{t}=\frac{\beta}{1+\beta \gamma} E_{t}\left[\hat{\pi}_{t+1}\right]+\frac{\gamma}{1+\beta \gamma} \hat{\pi}_{t-1}+\frac{\kappa}{1+\beta \gamma} \hat{m} c_{t}+e_{t}
$$

where $\hat{\pi}_{t}=\pi_{t}-\bar{\pi}$ is the inflation gap defined by the difference between the inflation rate and steady sate inflation $\bar{\pi}$ (potentially the inflation target set by the central bank) and $m c_{t}$ is marginal cost. This hybrid NKPC can be derived with a full price indexation to trend inflation and is widely used in the literature (e.g. Smets and Wouters (2007)). When considering a reduced form regression from this model, a change in the trend inflation can be associated with a structural break in the unconditional mean of inflation and a change in the degree of price stickiness produces a shift in persistence of inflation dynamics. Also, a change in the residual variance can be interpreted as different sizes of economic shocks.

5 Giordani and Kohn (2008) consider an alternative model which allows structural breaks to come through mixture distributions in state innovations and shifts in variance occur independently of shifts in other parameters. However, this specification still cannot distinguish breaks in the unconditional mean from shifts in persistence. 
of structural change are determined by a Bayesian model selection procedure using Bayes factors. For robustness, I also calculate marginal probabilities for the number of structural breaks in each individual parameter group because the model selection procedure using the Bayes factors chooses only one model.

I employ the modified approach to an autoregressive model for postwar US GDP deflator inflation and CPI inflation by allowing for shifts in the unconditional mean, a group of persistence parameters, and/or the residual variance. The Bayesian model selection procedure shows distinct patterns of structural changes from two different measures of inflation. CPI inflation experienced a dramatic drop in persistence around the early 1980s, but GDP deflator inflation is still persistent. Also, the residual variance for both inflation measures switched from a low volatility regime to a high volatility regime in the early 1970s, but it returned to another low volatility regime at different dates: the early 1980s for GDP deflator inflation and the early 1990s for CPI inflation. The residual variance for CPI inflation has increased again since the early 2000s, while GDP deflator inflation has remained less volatile. This different pattern of changes in the variance is also confirmed using unobserved components model with stochastic volatility in Stock and Watson (2007). However, I do not find evidence of a structural shift in the unconditional mean. The marginal probability calculations for the number of breaks in each individual parameter group also strongly support the model selection results based on the Bayes factors. The structural breaks at different dates reflect the possibility that parameter shifts might be caused by a variety of factors.

The remainder of this paper describes the methods and then discusses the empirical results and how they compare to existing results in the literature.

\section{Model specification and Bayesian inference}

\subsection{Model specification}

I consider a $p$ th-order autoregressive model for an analysis of inflation dynamics and the AR(p) model allows for structural breaks in three parameter groups such as (i) the unconditional mean $\mu$, (ii) persistence $\left(\phi_{1}, \ldots\right.$, $\phi_{p}$ ) and (iii) the residual variance $\sigma^{2}$. I focus on changes in the unconditional mean rather than the intercept because changes in the intercept may contain mixed information on changes in level and persistence. Sims (2001) and Stock (2001) note that allowing for changes in the residual variance helps avoid any potential distortions of identifying structural breaks in coefficients. ${ }^{6}$ The AR(p) model with structural breaks is given by

$$
\pi_{t}=\mu_{S_{1, t}}+\phi_{1, S_{2, t}}\left(\pi_{t-1}-\mu_{S_{1, t-1}}\right)+\cdots+\phi_{p, S_{2, t}}\left(\pi_{t-p}-\mu_{S_{1, t-p}}\right)+e_{t}, e_{t} \sim N\left(0, \sigma_{S_{3, t}}^{2}\right)
$$

where $S_{1, t} \in\left\{1, \ldots, M_{1}+1\right\}, S_{2, t} \in\left\{1, \ldots, M_{2}+1\right\}$, and $S_{3, t} \in\left\{1, \ldots, M_{3}+1\right\}$ represent the regimes for the unconditional mean parameter with $M_{1}$ breaks, the persistence parameter group with $M_{2}$ breaks and the residual variance with $M_{3}$ breaks, respectively. The nature of the structural breaks in each parameter group is independent of one another in terms of the number and timing of structural changes.

To make inferences about multiple-group changepoint models, I modify Chib's (1998) approach in which the structural breaks are interpreted as regime transitions. Chib (1998) assumes that all the parameters which undergo the structural changes have the structural shifts at the same dates. ${ }^{7}$ Thus, I propose an efficient Bayesian MCMC method that allows for a number of possibilities for the nature of structural breaks. This modified approach is developed to have the following attractive features: (i) model specification of considering multiple structural changes in multiple parameters; (ii) model flexibility in allowing the multiple

6 In a similar spirit, Sims and Zha (2006) find that the best fitting vector autoregressive model does not require changes in coefficients when regime switches in disturbance variances are allowed.

7 This type of model can be called a single-group changepoint model (e.g. both pure and partial structural change models in Bai and Perron 2003). 
structural breaks to occur mutually independently at different dates; and (iii) model selection procedure by comparing various potentially non-nested structural break models.

Suppose a multiple-group changepoint model which allows parameters to change at different dates with the different number of breaks. ${ }^{8}$ For example, in (1) the model for inflation dynamics may have one break in the unconditional mean, two breaks in persistence, and three breaks in the residual variance. In this case, the modified approach would require only augmentations with three independent latent regime indicator variables $\left(\tilde{S}_{1, T}, \tilde{S}_{2, T}, \tilde{S}_{3, T}\right)$, where $\tilde{S}_{i, T}=\left[S_{i, 1} S_{i, 2} \ldots S_{i, T-1} S_{i, T}\right]^{\prime}$ for each parameter group $i=1,2$, 3, and three transition probability matrices $\left(P_{1}, P_{2}, P_{3}\right)$ corresponding to three parameter groups while the single-group changepoint model is augmented with only one regime indicator variable and one transition probability matrix. Since all the regime indicator variables are mutually independent in the multiple-group changepoint model, the date of regime transition in a parameter is allowed to occur close to that of regime transition in other parameters without any necessary minimum distance unlike the restriction in Levin and Piger (2007).

For a parameter group $i$, the latent state variable $S_{i, t}$ follows a first-order Markov process with the transition probabilities constrained: for $k=1, \ldots, M_{i}$

$$
\operatorname{Pr}\left[S_{i, t}=k \mid S_{i, t-1}=k\right]=p_{k, k}^{i} \text { and } \operatorname{Pr}\left[S_{i, t}=k+1 \mid S_{i, t-1}=k\right]=p_{k, k+1}^{i}=1-p_{k, k}^{i}
$$

and

$$
\operatorname{Pr}\left[S_{i, t}=M_{i}+1 \mid S_{i, t-1}=M_{i}+1\right]=1 \text { for the last regime } M_{i}+1
$$

where $p_{k, k}^{i}$ indicates the probability that a regime $S_{i, t}$ for the parameter group $i$ stays in the current regime $k$. A transition probability matrix $P_{i}$ for the parameter group $i$ can then be formed as a $\left(M_{i}+1\right)$-by- $\left(M_{i}+1\right)$ matrix with elements containing information about the first-order Markov process in (2) and (3). ${ }^{9}$

A joint posterior density can be obtained as being proportional to a product of a prior density and a likelihood function of $Y_{T}=\left[y_{1} \ldots y_{T}\right]^{\prime}$ such as

$$
\pi\left(\boldsymbol{\theta}, \boldsymbol{P} \mid Y_{T}\right) \propto \pi(\boldsymbol{\theta}, \boldsymbol{P}) f\left(Y_{T} \mid \boldsymbol{\theta}, \boldsymbol{P}\right)
$$

where $\pi(\cdot)$ denotes a density function; $\theta=\left(\tilde{\mu}, \tilde{\phi}, \tilde{\sigma}^{2}\right)$ is a collection of model parameters; $\tilde{\mu}=\left(\mu_{1}, \ldots, \mu_{M_{1}+1}\right), \tilde{\phi}=\left(\phi_{1,1}, \ldots, \phi_{p, 1}, \ldots, \phi_{1, M_{2}+1}, \ldots, \phi_{p, M_{2}+1}\right)$, and $\tilde{\sigma}^{2}=\left(\sigma_{1}^{2}, \ldots, \sigma_{M_{3}+1}^{2}\right)$; and $\boldsymbol{P}=\left(P_{1}, P_{2}, P_{3}\right)$ is a collection of transition probability matrices. The model parameters, $\theta=\left(\tilde{\mu}, \tilde{\phi}, \tilde{\sigma}^{2}\right)$, are then augmented with the transition probability matrices, $\boldsymbol{P}=\left(P_{1}, P_{2}, P_{3}\right)$, and the latent regime indicators $\left(\tilde{S}_{1, T}, \tilde{S}_{2, T}, \tilde{S}_{3, T}\right)$. The MCMC sampler is developed through a hierarchical specification in which one draws the model parameters conditional on the regime indicators and the observed data; the regime indicators conditional on the model parameters and the observed data; and finally the transition probabilities conditional on the regime indicators via Gibbs sampling. More details for the MCMC sampling algorithm are explained in the Appendix.

8 Pesaran, Pettenuzzo and Timmermann (2006) and Koop and Potter (2007) extend Chib’s (1998) approach to improve forecasting ability and Giordani and Kohn (2008) take a different approach of mixture models for making inference about structural breaks. The multiple-group changepoint approach may be also evaluated in terms of forecasting performance. However, Bauwens et al. (2015) recently examine the forecasting performance of various structural break models including approaches based on Pesaran, Pettenuzzo and Timmermann (2006), Koop and Potter (2007), and Giordani and Kohn (2008) and they find that there is no one single method which can be recommended universally.

9 This restriction can be expressed in matrix form where $p_{j, k}$ is placed in the $(j, k)$ th entry of the transition matrix

$$
P=\left[\begin{array}{ccccc}
p_{1,1} & p_{1,2} & 0 & \cdots & 0 \\
0 & p_{2,2} & p_{2,3} & \cdots & 0 \\
\vdots & \vdots & \vdots & \vdots & \vdots \\
\cdots & \vdots & 0 & p_{M, M} & p_{M, M+1} \\
0 & 0 & \cdots & 0 & 1
\end{array}\right] .
$$

I drop the superscript $i$ for notational simplicity. 


\subsection{Model selection procedures}

I consider two different model selection procedures: (i) the Bayes factor comparison using the marginal likelihoods and (ii) the posterior probability for the number of structural breaks in each individual parameter group by integration. Thus, I find not only the most preferred model based on the Bayes factor but also the marginal probability for the number of breaks in each parameter group for robustness to the model selection results. In this analysis, the maximum number of structural breaks is specified to four for each parameter group and in total, 125 different models for each inflation measure are considered including a model with no break $\left(125=5^{3}\right)$.

The Bayes factor is presented in favor of the alternative model, $\mathcal{M}=\left(M_{1}, M_{2}, M_{3}\right)$ versus the most preferred model, $\mathcal{M}^{*}=\left(M_{1}^{*}, M_{2}^{*}, M_{3}^{*}\right)$ by

$$
B F=\frac{m\left(Y_{T} \mid \mathcal{M}=\left(M_{1}, M_{2}, M_{3}\right)\right)}{m\left(Y_{T} \mid \mathcal{M}^{*}=\left(M_{1}^{*}, M_{2}^{*}, M_{3}^{*}\right)\right)}
$$

where $m\left(Y_{T} \mid \mathcal{M}=\left(M_{1}, M_{2}, M_{3}\right)\right)$ is the marginal likelihood for the model with structural breaks of $\left(M_{1}, M_{2}, M_{3}\right)$. I calculate the marginal likelihood at the posterior mean. The algorithm to compute the marginal likelihood is described in the Appendix.

I also calculate the posterior probability for the number of structural breaks in each individual parameter group by integrating out the number of breaks in other parameters. For example, the posterior probability for $l$ structural breaks in the parameter $\tilde{\mu}$, denoted by $M_{1}=l$, is given by

$$
\operatorname{Pr}\left(M_{1}=l \mid Y_{T}\right)=\frac{m\left(Y_{T} \mid M_{1}=l\right) \pi\left(M_{1}=l\right)}{\sum_{i=0}^{4} m\left(Y_{T} \mid M_{1}=i\right) \pi\left(M_{1}=i\right)}
$$

where

$$
m\left(Y_{T} \mid M_{1}=l\right)=\sum_{j=0}^{4} \sum_{k=0}^{4} m\left(Y_{T} \mid M_{1}=l, M_{2}=j, M_{3}=k\right) \pi\left(M_{2}=j, M_{3}=k \mid M_{1}=l\right)
$$

is the integrated likelihood when $M_{1}=l ; \pi\left(M_{1}=l\right)$ is the prior probability for $M_{1}=l$; and $\pi\left(M_{2}=j, M_{3}=k \mid M_{1}=l\right)$ is the joint prior probability for $M_{2}=j$ and $M_{3}=k$ conditional on $M_{1}=l$. Because all the models are considered $a$ priori equally likely as well as independent, $\pi\left(M_{1}=l\right)$ is equal to $1 / 5$ and $\pi\left(M_{2}=j, M_{3}=k \mid M_{1}=l\right)$ is equal to $1 / 25$ when the maximum number of structural breaks is specified to four as in this analysis. In fact, the posterior probability of $l$ structural breaks in the parameter $\tilde{\mu}$ is simply the sum of posterior probabilities for all the models such that $M_{1}=l$. For other parameters, $\tilde{\phi}$ and $\tilde{\sigma}^{2}, \operatorname{Pr}\left(M_{2}=j \mid Y_{T}\right)$ and $\operatorname{Pr}\left(M_{3}=k / Y_{T}\right)$ can be easily obtained by using the same approach in (5). Note that all the terms in (5) are readily available when the marginal likelihood calculations are completed.

\section{Structural breaks in inflation dynamics}

\subsection{Data and prior}

I consider two different quarterly US inflation measures based on the consumer price index (CPI) and the GDP deflator. ${ }^{10}$ Each inflation is defined as 100 times the log change in the corresponding price index for the period of 1953:Q1-2013:Q4.

Figure 1 depicts the two inflation series. Both inflation measures appear to be less volatile for the "Great Moderation" period since the early 1980s but CPI inflation has been recently more volatile. Moreover, CPI inflation appears to be less persistent than GDP deflator inflation. Table 1 presents summary statistics over

10 I use the CPI series for all urban consumers (CPIAUCSL) and the GDP implicit price deflator (GDPDEF) from the Federal Reserve Economic Database (FRED). They are all seasonally adjusted. 


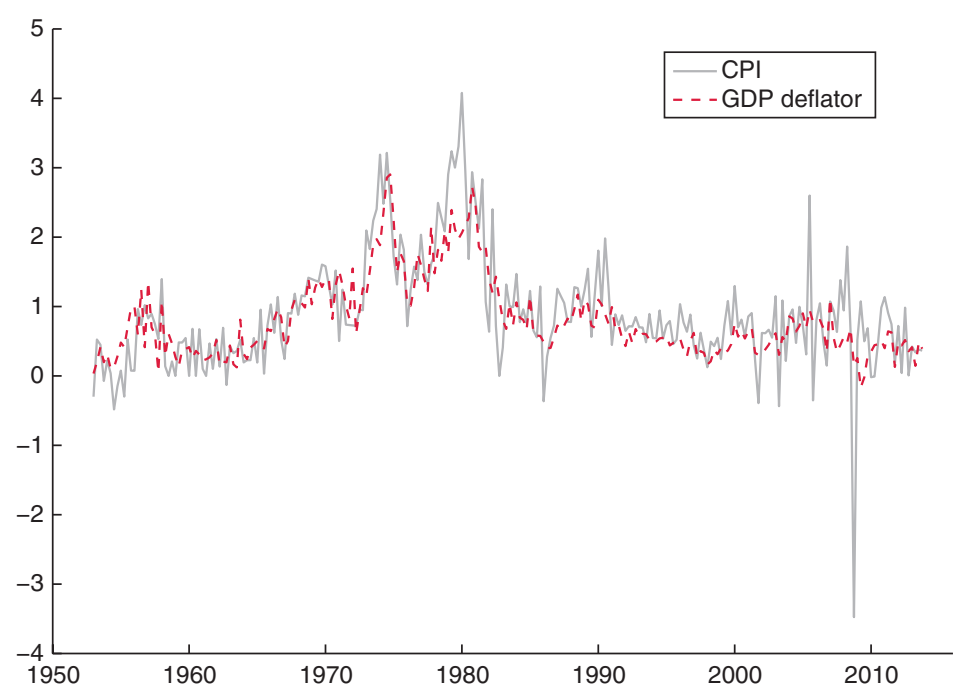

Figure 1: US inflation rates: CPI and GDP deflator (quarterly percentage change): 1953:Q1-2013:Q4.

Table 1: Summary statistics for inflation series: 1953:Q1-2013:Q4.

\begin{tabular}{lrrr}
\hline Series & Mean & SD & First order autocorr. \\
\hline CPI & 0.89 & 0.81 & 0.61 \\
GDP & 0.80 & 0.57 & 0.86 \\
Corr & 0.78 & & \\
\hline
\end{tabular}

CPI refers to CPI inflation and GDP refers to GDP deflator inflation.

the sample period. CPI inflation has a slightly higher mean, is less persistent, and more volatile than for GDP deflator inflation. In addition, the correlation between the two inflation measures is 0.78 . Thus, the timing and the number of structural changes might be different across model parameters such as the unconditional mean, persistence, and the residual variance as well as across the different measures of inflation. In this case, the multiple-group changepoint model can effectively detect the structural changes in individual parameter groups.

In this analysis, the lag order $p$ is set to two for both series by the Bayesian information criterion. The diffuse and same prior distributions across different regimes are chosen in order to avoid any distortions from the choice of specific prior distributions when estimating different structural break models. The priors for regression coefficients are distributed with mean zero and variance one (i.e., $\left.\mu, \phi_{i} \sim \mathcal{N}(0,1)\right)$ and the priors of variance parameters follow an Inverse Gamma distribution such as $\sigma^{2} \sim I G\left(\frac{5.0}{2}, \frac{1.5}{2}\right)$ for CPI inflation and $\sigma^{2} \sim I G\left(\frac{4.2}{2}, \frac{0.5}{2}\right)$ for GDP deflator inflation. The priors for the variance parameters are set differently reflecting the fact that GDP deflator inflation is more persistent and less volatile than CPI inflation so that the residual variance for the GDP inflation regression is much smaller than for the CPI inflation regression. See the summary statistics in Table 1 . The prior of the transition probability that the current regime $k$ stays in the same regime $k$ in the next period is distributed as $p_{k k}-\operatorname{Beta}(10,0.1)$. The prior expected duration of a given regime is about 101 quarters and the prior expected number of breaks is 2.4 given the sample size of 241 . All the estimations are based on 3000 Gibbs simulations after discarding 3000 burn-ins. ${ }^{11}$

11 Estimating multiple-group changepoint models does not require considerable computational time. For example, the most preferred model for CPI inflation with $\mathcal{M}^{*}=\left(M_{1}^{*}=0, M_{2}^{*}=1, M_{3}^{*}=3\right)$ runs about $3 \mathrm{~min} 2.78 \mathrm{~s}$ using Intel Core i7-4770 CPU. The code is written and run in GAUSS 10. 


\subsection{Empirical findings for CPI inflation}

I first discuss model selection results for CPI inflation. Table 2 presents the ten best models based on marginal likelihood calculations among 125 models. The comparison of the marginal likelihoods shows that the most preferred model has one structural break in persistence, three structural breaks in the residual variance, and no structural break in the unconditional mean. The most preferred model clearly dominates the other models in the sense that the Bayes factor is lower than $1 / 8$ in favor of any alternative model.

Table 3 lists the marginal posterior probability of the number of structural breaks in individual parameter groups using (5). The highest probability of the number of breaks for each parameter is calculated as follows: 0.89 for no break in the unconditional mean, 0.97 for one break in persistence, and 0.91 for three breaks in the residual variance. This finding from the marginal probability calculations is completely consistent with the model selection of $\mathcal{M}^{*}=\left(M_{1}^{*}=0, M_{2}^{*}=1, M_{3}^{*}=3\right)$ based on the Bayes factors.

Table 4 summarizes the posterior distributions with mean and standard deviation and Figure 2 plots the posterior mean and $90 \%$ credible band for each parameter group over the sample period. The sum of autoregressive coefficients is used for the measure of persistence. The posterior distributions clearly

Table 2: US CPI inflation: model selection.

\begin{tabular}{lrr}
\hline Model (\# of breaks) $\mathcal{M}=\left(\boldsymbol{M}_{\mathbf{1}}, \boldsymbol{M}_{\mathbf{2}}, \boldsymbol{M}_{\mathbf{3}}\right)$ & Log marginal likelihood & Bayes factor \\
\hline$(0,1,3)$ & -203.55 & 1.0000 \\
$(1,1,3)$ & -205.66 & 0.1206 \\
$(0,1,4)$ & -206.17 & 0.0728 \\
$(0,2,3)$ & -207.36 & 0.0221 \\
$(0,1,2)$ & -207.46 & 0.0200 \\
$(0,0,3)$ & -207.85 & 0.0135 \\
$(0,1,1)$ & -208.16 & 0.0099 \\
$(1,1,4)$ & -208.35 & 0.0082 \\
$(2,1,3)$ & -208.77 & 0.0054 \\
$(1,1,2)$ & -209.04 & 0.0041 \\
\hline
\end{tabular}

$M_{1}, M_{2}$, and $M_{3}$ denote the number of breaks in the unconditional mean $(\mu)$, the persistence $\left(\phi_{1}, \phi_{2}\right)$, and the residual variance

$\left(\sigma^{2}\right)$, respectively. Bayes factors are calculated in favor of the alternative model: $B F=\frac{m\left(Y_{T} \mid \mathcal{M}_{\mathcal{A}}\right)}{m\left(Y_{T} \mid \mathcal{M}^{*}=(0,1,3)\right)}$.

Table 3: US CPI inflation: posterior probability of the number of structural breaks in individual parameter group.

\begin{tabular}{lrr}
\hline Parameter & \# of breaks & Posterior probability \\
\hline Uncond. mean & 0 & $\mathbf{0 . 8 9}$ \\
& 1 & 0.11 \\
& 2 & 0.00 \\
& 3 & 0.00 \\
Persistence & 4 & 0.00 \\
& 0 & 0.01 \\
& 1 & $\mathbf{0 . 9 7}$ \\
& 2 & 0.02 \\
Residual variance & 3 & 0.00 \\
& 4 & 0.00 \\
& 0 & 0.00 \\
& 1 & 0.01 \\
& 2 & 0.02 \\
& 3 & $\mathbf{0 . 9 1}$ \\
\hline
\end{tabular}

Bold values indicate the highest posterior probability for individual parameter groups. 
Table 4: US CPI inflation: posterior distributions for the most preferred model [\# of breaks $\left.\left(M_{1}^{*}, M_{2}^{*}, M_{3}^{*}\right)=(0,1,3)\right]$.

\begin{tabular}{|c|c|c|c|c|c|}
\hline \multirow[t]{2}{*}{ Parameter } & \multirow[t]{2}{*}{ Prior } & \multicolumn{4}{|r|}{ Posterior } \\
\hline & & Regime1 & Regime2 & Regime3 & Regime4 \\
\hline \multirow[t]{2}{*}{$\mu$} & 0.000 & 0.686 & & & \\
\hline & $(1.000)$ & $(0.070)$ & & & \\
\hline \multirow{2}{*}{$\phi_{1}$} & 0.000 & 0.436 & 0.086 & & \\
\hline & $(1.000)$ & $(0.085)$ & $(0.126)$ & & \\
\hline \multirow[t]{2}{*}{$\phi_{2}$} & 0.000 & 0.458 & 0.013 & & \\
\hline & $(1.000)$ & $(0.085)$ & $(0.116)$ & & \\
\hline \multirow[t]{2}{*}{$p_{2, i i}$} & 0.990 & 0.992 & & & \\
\hline & $(0.030)$ & $(0.008)$ & & & \\
\hline \multirow[t]{2}{*}{$\sigma^{2}$} & 0.500 & 0.143 & 0.427 & 0.112 & 0.678 \\
\hline & $(0.707)$ & $(0.025)$ & $(0.103)$ & $(0.036)$ & $(0.149)$ \\
\hline \multirow{2}{*}{$p_{3, i i}$} & 0.990 & 0.987 & 0.986 & 0.981 & \\
\hline & $(0.030)$ & $(0.012)$ & $(0.014)$ & $(0.018)$ & \\
\hline
\end{tabular}

Standard deviations are reported in parentheses.

A

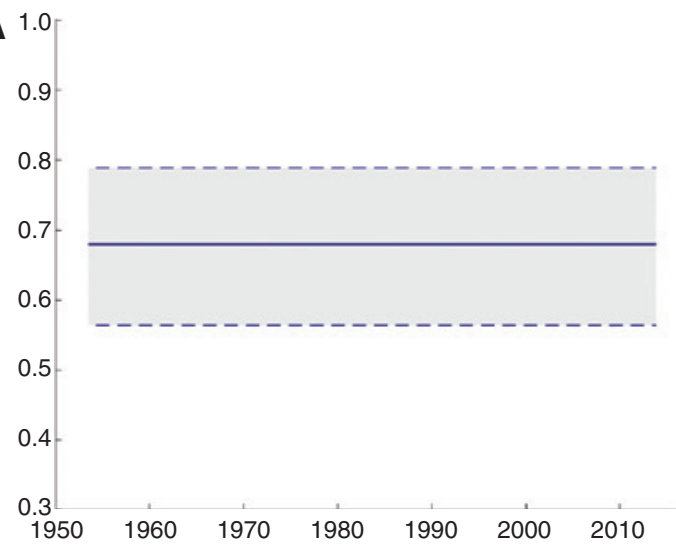

B 1.2

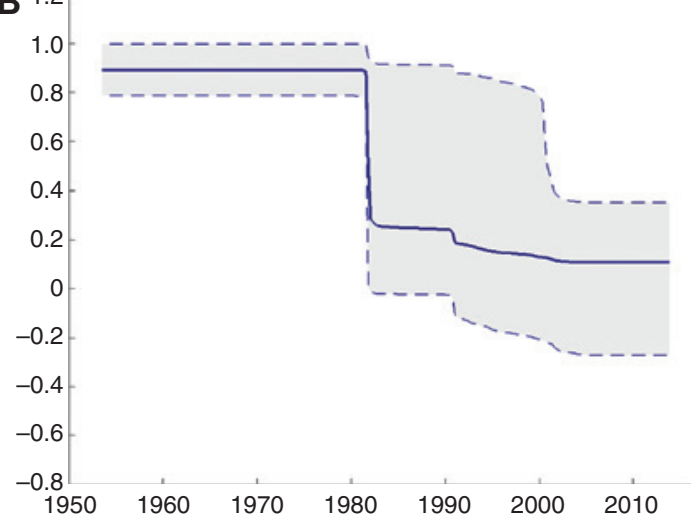

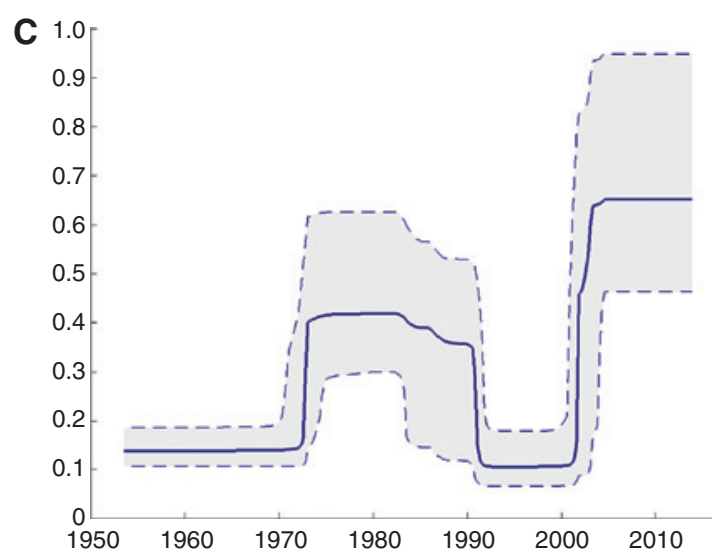

Figure 2: US CPI inflation: posterior distribution of parameters over time.

(A) Unconditional mean, (B) persistence (sum of AR parameters), (C) residual variance.

Posterior mean and $90 \%$ band are plotted over time. The estimated break dates (posterior mode) are 1981:Q3 for persistence and 1972:Q4, 1991:Q1, and 2001:Q2 for the residual variance.

show that persistence sharply decreased from 0.89 to 0.10 (posterior mean) in the early 1980s while the residual variance switched from a low volatility regime to a high volatility regime around the early 1970 , returned to another low volatility regime around the early 1990s, and then has increased again since the 
early 2000s. ${ }^{12}$ The changes in the residual variance also appear to be abrupt. It is evident that the break point for persistence is different from the break points for the residual variance. Thus, these structural changes could be caused by different sources.

\subsection{Empirical findings for GDP deflator inflation}

Now, I apply the modified approach to an AR(2) model for GDP deflator inflation. Table 5 presents calculations for the marginal likelihood and Bayes factor. The marginal likelihood calculations select the model with two breaks in the residual variance only. The Bayes factor between $\mathcal{M}^{*}=(0,0,2)$ and $\mathcal{M}=(0,0,1)$, the second preferred model, is 0.61 . Table 6 shows the marginal probability for the number of breaks in each parameter group. The posterior probability for two breaks in the residual variance $\left(\sigma^{2}\right)$ is 0.60 and for one break is 0.37 while the posterior probabilities for no break in the unconditional mean $(\mu)$ and in persistence $(\phi)$ are 0.99

Table 5: US GDP deflator inflation: model selection.

\begin{tabular}{lrr}
\hline Model (\# of breaks) $\mathcal{M}=\left(\boldsymbol{M}_{\mathbf{1}}, \boldsymbol{M}_{\mathbf{2}}, \boldsymbol{M}_{\mathbf{3}}\right)$ & Log marginal likelihood & Bayes factor \\
\hline$(0,0,2)$ & -23.30 & 1.0000 \\
$(0,0,1)$ & -23.80 & 0.6105 \\
$(0,1,2)$ & -26.18 & 0.0561 \\
$(0,0,3)$ & -26.42 & 0.0441 \\
$(0,1,1)$ & -26.77 & 0.0311 \\
$(1,0,1)$ & -27.88 & 0.0103 \\
$(1,0,2)$ & -27.98 & 0.0093 \\
$(0,1,3)$ & -29.14 & 0.0029 \\
$(0,0,4)$ & -29.73 & 0.0016 \\
$(0,2,2)$ & -30.02 & 0.0012 \\
\hline
\end{tabular}

For the proposed approach, $M_{1}, M_{2}$, and $M_{3}$ denote the number of breaks in the unconditional mean $(\mu)$, the persistence $\left(\phi_{1}, \phi_{2}\right)$, and the residual variance $\left(\sigma^{2}\right)$, respectively. Bayes factors are calculated in favor of the alternative model: $B F=\frac{m\left(Y_{T} \mid \mathcal{M}_{\mathcal{A}}\right)}{m\left(Y_{T} \mid \mathcal{M}^{*}=(0,0,2)\right)}$.

Table 6: US GDP deflator inflation: posterior probability of the number of structural breaks in individual parameters.

\begin{tabular}{lrr}
\hline Parameter & \# of breaks & Posterior probability \\
\hline Uncond. mean & 0 & $\mathbf{0 . 9 9}$ \\
& 1 & 0.01 \\
& 2 & 0.00 \\
& 3 & 0.00 \\
Persistence & 4 & 0.00 \\
& 0 & $\mathbf{0 . 9 5}$ \\
& 1 & 0.05 \\
& 2 & 0.00 \\
Residual variance & 3 & 0.00 \\
& 4 & 0.00 \\
& 0 & 0.00 \\
& 1 & 0.37 \\
& 2 & $\mathbf{0 . 6 0}$ \\
& 3 & 0.03 \\
\hline
\end{tabular}

Bold values indicate the highest posterior probability for individual parameter groups.

12 Giordani and Kohn (2008) consider an AR(1) model of CPI inflation for the sample period of 1951-2004 and also find a structural break in the early 1990s rather than the mid 1980s, the so-called “Great Moderation” breakpoint. 
and 0.95 , respectively. Thus, not only the marginal likelihood comparisons but also the posterior probability calculations produce very strong evidence that the autoregressive model for GDP deflator inflation has no break in the unconditional mean and persistence but the specification for two breaks in the residual variance is slightly more preferred than for one break. ${ }^{13}$ This result is also consistent with the finding that evidence for shifts in persistence for GDP deflator inflation is not statistically significant, particularly once allowing for shifts in the residual variance as in Pivetta and Reis (2007) and Stock (2002).

Table 7 summarizes posterior distributions for the parameters in the most preferred model and Figure 3 plots posterior mean and 90\% credible band for each parameter group. The residual variance switched from a low volatility regime to a high volatility regime around the early 1970s and then returned to another low volatility regime around the early 1980s. In addition, the residual variance for the first regime appears to be bigger than that for the third regime. The changes in the residual variance are also abrupt and this implies that the multiple-group changepoint model provides precise information about the timing of the structural breaks in the residual variance. Note also that GDP deflator inflation is highly persistent in the sense that the sum of the autoregressive coefficients (posterior mean) is 0.88 . This finding on high inflation persistence is consistent with that in the literature (e.g. Fuhrer and Moore (1995)). However, the 90\% credible band does not cover the unit root. ${ }^{14}$

\section{Robustness}

\subsection{Comparison with Chib’s (1998) approach}

For robustness, I consider Chib's approach, a single-group changepoint model, in which all the parameters including the residual variance undergo breaks at the same time. The models are estimated using the same priors as in the multiple-group changepoint models. The results for the Bayes factor calculations are summarized for both measures of inflation in Table 8. Based on Chib's approach, the values of the highest $(\log )$

Table 7: US GDP deflator inflation: posterior distributions for the most preferred model [\# of breaks $\left(M_{1}^{*}, M_{2}^{*}, M_{3}^{*}\right)=(0,0,2)$ ].

\begin{tabular}{lrrrr}
\hline Parameter & Prior & & & Posterior \\
\cline { 3 - 4 } & & Regime 1 & Regime 2 & Regime 3 \\
\hline$\mu$ & 0.000 & 0.611 & & \\
& $(1.000)$ & $(0.205)$ & & \\
$\phi_{1}$ & 0.000 & 0.494 & & \\
& $(1.000)$ & $(0.064)$ & & 0.038 \\
$\phi_{1}$ & 0.000 & 0.387 & 0.174 & $(0.005)$ \\
$\sigma^{2}$ & $(1.000)$ & $(0.062)$ & $(0.041)$ & 0.980 \\
$p_{3, i i}$ & 0.227 & 0.078 & $(0.017)$ & \\
& $(0.719)$ & $(0.014)$ & 0.985 & \\
\hline
\end{tabular}

Standard deviations are reported in parentheses.

13 The break date for the autoregressive model with one break in the residual variance is consistent with the second break date around the early 1980s for the model with two breaks. As shown in Table 7, the difference in the residual variances is more pronounced in the second break.

14 I have conducted robustness checks by doubling the standard deviations of priors as well as considering an AR(4) model. I found that the posterior distributions and the timing of the structural breaks are broadly identical and they are robust to the alternative specifications. Also, additional coefficients for higher-order lags in the AR(4) models appear to be insignificant. 


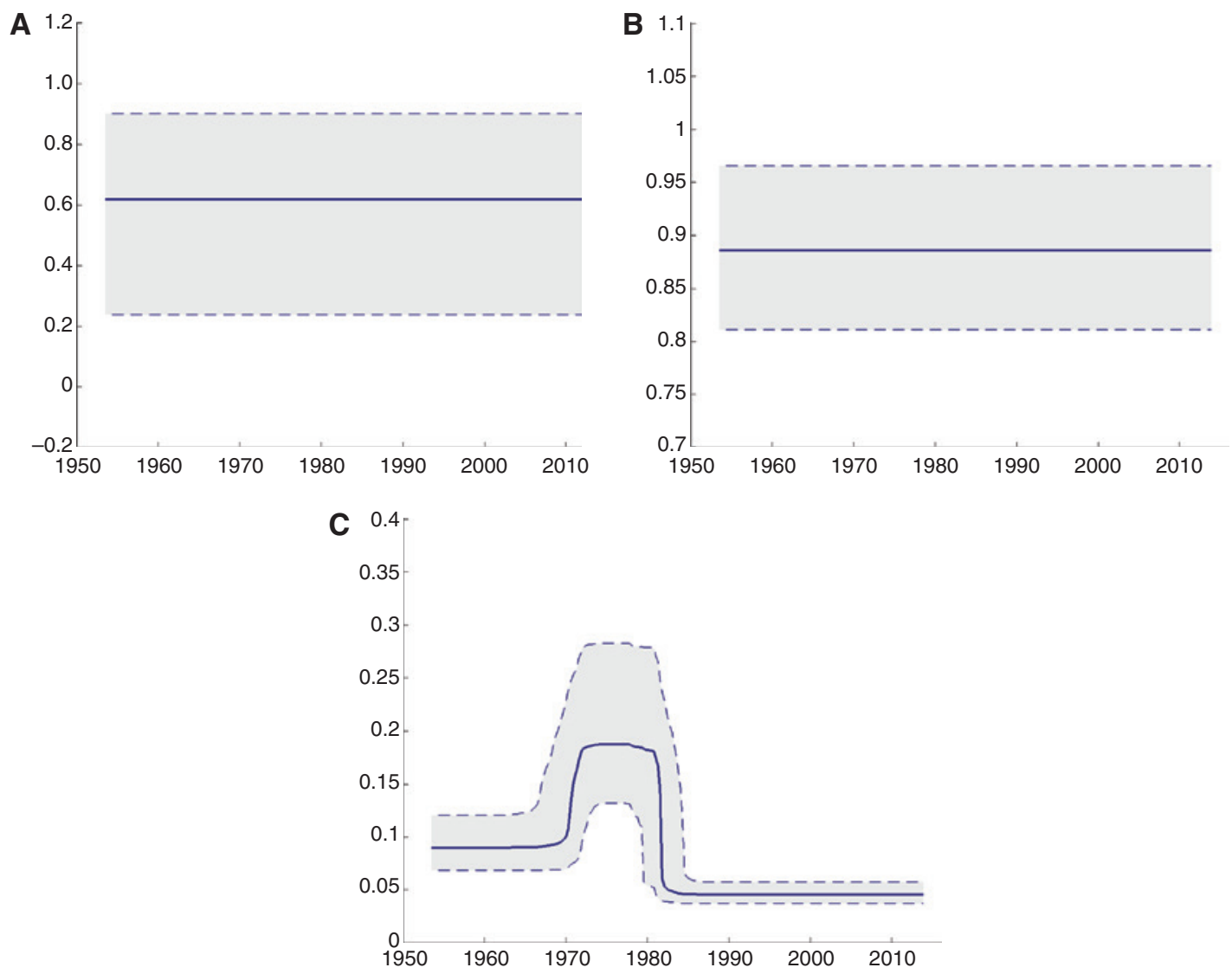

Figure 3: GDP deflator inflation: posterior distribution of parameters over time.

(A) Unconditional mean, (B) persistence (Sum of AR parameters), (C) residual variance.

Posterior mean and 90\% band are plotted over time. The estimated break dates (posterior mode) are 1970:Q2 and 1981:Q2 for the residual variance.

Table 8: Comparison to Chib's (1998) approach: model selection.

\begin{tabular}{lrr}
\hline Model (\# of breaks) & Log marginal likelihood & Bayes factor \\
\hline (a) CPI inflation & & \\
0 & -233.43 & 0.0000 \\
1 & -212.13 & 0.0002 \\
2 & -211.67 & 0.0003 \\
3 & -208.54 & 0.0068 \\
4 & -215.94 & 0.0000 \\
(b) GDP deflator inflation & & \\
0 & -34.61 & 0.0000 \\
1 & -31.43 & 0.0003 \\
2 & -32.55 & 0.0001 \\
3 & -39.22 & 0.0000 \\
4 & -49.25 & 0.0000 \\
\hline
\end{tabular}

Bayes factors are calculated in favor of the alternative model: $B F=\frac{m\left(Y_{T} \mid \mathcal{M}_{\mathcal{A}}\right)}{m\left(Y_{T} \mid \mathcal{M}^{*}\right)}$ for each measure of inflation. $m\left(Y_{T} \mid \mathcal{M}^{*}\right)$ is the most preferred model based on the multiple-group changepoint approach for each measure of inflation.

marginal likelihood are equal to -208.54 (three breaks) for CPI inflation and -31.43 (one break) for GDP deflator inflation, respectively. However, these marginal likelihood values are significantly lower than those based on the multiple-group changepoint approach. 
I also consider another modified approach in which the unconditional mean and persistence parameters (i.e. conditional mean) undergo co-breaks at the same time, which are independent of the breaks in the residual variance. I estimate the model with one break in the conditional mean and three breaks in the residual variance in accordance with the empirical finding in the previous section. The log marginal likelihood for this modified model is calculated to be -204.81 , which is lower than that for the most preferred model $(-203.55)$ based on the multiple-group changepoint approach. The Bayes factor is then equal to 0.28 (alternatively 3.53 in favor of the most preferred model, $\left.\mathcal{M}^{*}=(0,1,3)\right)$, which shows substantial evidence for one break in persistence and three breaks in the residual variance when adopting Jeffreys's (1961) interpretation. ${ }^{15}$ Note that the most preferred model for GDP deflator inflation has no break in the unconditional mean and persistence. Therefore it is not required to estimate the modified model additionally.

\subsection{Unobserved components models with stochastic volatility}

The empirical results reported in Section 3 show that there have been large changes in the variance, but two measures of inflation exhibit the different evolution of the residual variance in terms of the number and timing of the breaks. Following Stock and Watson (2007), I consider an unobserved components model with stochastic volatility (UC-SV) and further examine whether these empirical findings on the shifts in the residual variance are robust. ${ }^{16}$

Stock and Watson (2007) estimate an UC-SV model for GDP deflator inflation from 1953:Q1 to 2004:Q4. They find that there have been substantial movements over time in the standard deviation of the permanent component while there is little change in the standard deviation of the transitory innovation. I examine CPI inflation in addition to GDP deflator inflation using the longer sample period of 1953:Q1-2013:Q4, which is used in Section 3, in comparison to Stock and Watson (2007). The UC-SV model specification is exactly the same as in Stock and Watson (2007):

$$
\begin{aligned}
& \pi_{t}=\tau_{t}+\eta_{t}, \text { where } \eta_{t}=\sigma_{\eta, t} \xi_{\eta, t} \\
& \tau_{t}=\tau_{t-1}+\epsilon_{t}, \text { where } \epsilon_{t}=\sigma_{\epsilon, t} \xi_{\epsilon, t} \\
& \ln \sigma_{\eta, t}^{2}=\ln \sigma_{\eta, t-1}^{2}+v_{\eta, t} \\
& \ln \sigma_{\epsilon, t}^{2}=\ln \sigma_{\epsilon, t-1}^{2}+v_{\epsilon, t}
\end{aligned}
$$

where $\xi_{t}=\left(\xi_{n, t}, \xi_{\epsilon, t}\right)^{\prime}$ is i.i.d. $N\left(0, I_{2}\right) ; v_{t}=\left(v_{\eta, t}, v_{\epsilon, t}\right)^{\prime}$ is i.i.d. $N\left(0, \gamma I_{2}\right) ; \xi_{t}$ and $v_{t}$ are independently distributed; and $\gamma$ determines the smoothness of the stochastic volatility process. The scalar parameter $\gamma$ is set to 0.2 as in Stock and Watson (2007) and the posterior distributions of stochastic volatility are estimated by MCMC. Figures 4 and 5 plot the smoothed estimates (posterior mean and 67\% band) of $\sigma_{\epsilon, t}^{2}$ and $\sigma_{\eta, t}^{2}$ from the UC-SV models for GDP deflator inflation and CPI inflation, respectively. The empirical results for GDP deflator inflation are similar to Stock and Watson's (2007) results. The standard deviation of the permanent disturbance was moderate from the mid 1950s through the early 1970s, it was large during the 1970s through the mid 1980s, and it declined sharply in the mid-1980s, whereas the volatility of the transitory disturbance appears to have remained stable. This low-high-low volatility pattern on the permanent innovations is consistent with the regime changes in the residual variance based on the multiple-group changepoint model in Section 3. Note also that the changepoint model analysis finds that GDP inflation has been highly persistent for the whole sample period and this high persistence for GDP deflator inflation implies that the evolution of inflation volatility would be mostly attributed to the permanent innovations. This conjecture is confirmed by the substantial changes in the standard deviations of the permanent innovations and the relatively stable volatility of the transitory innovations. In contrast to GDP deflator inflation, the volatility of the transitory innovations for CPI inflation shows substantial movements while the permanent innovations for CPI inflation exhibit a

15 I thank an anonymous referee for suggesting this robustness analysis. 16 I thank an anonymous referee for suggesting this robustness check. 

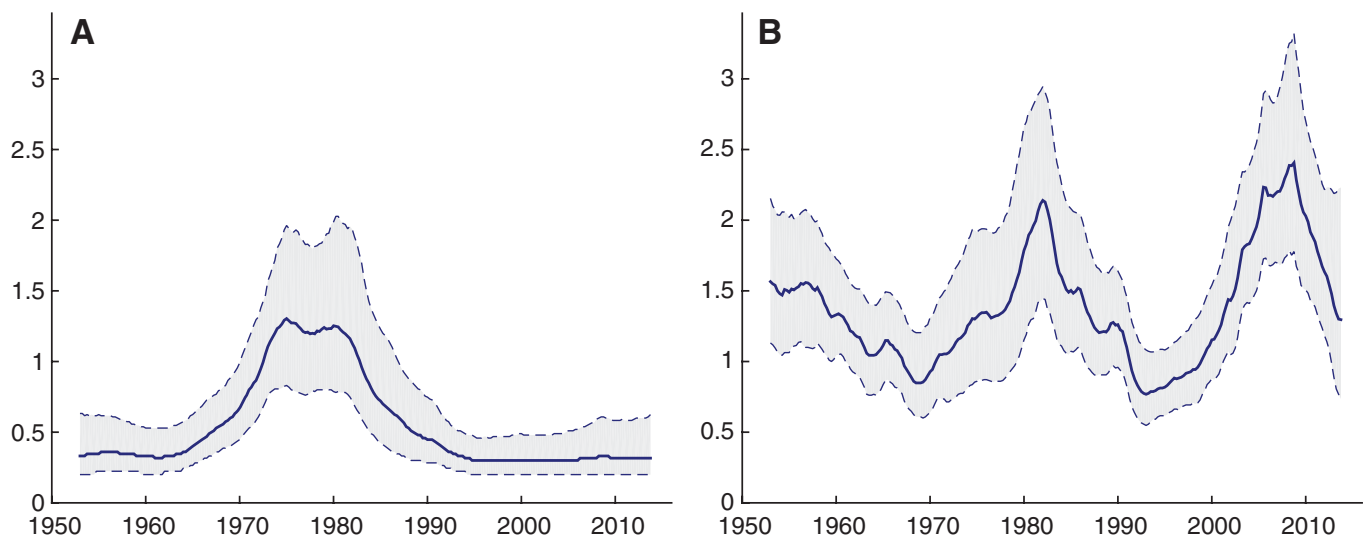

Figure 4: CPI inflation: stochastic volatility in UC-SV model. (A) Permanent innovations, (B) transitory innovations. Posterior mean and $67 \%$ band are plotted over time.
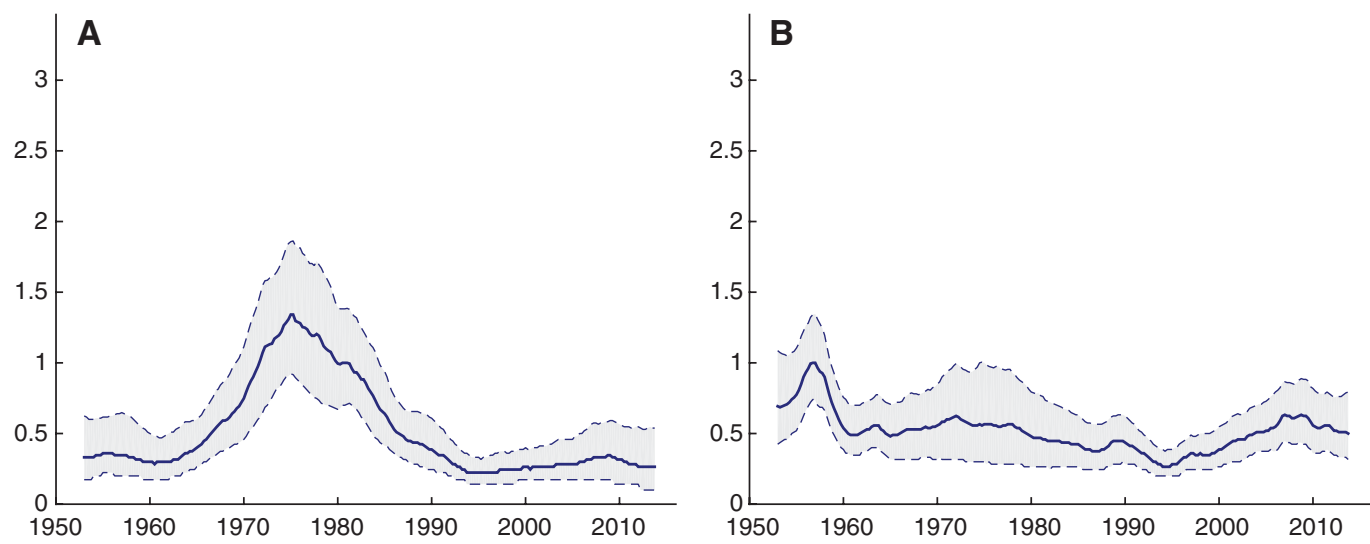

Figure 5: GDP deflator inflation: stochastic volatility in UC-SV model. (A) Permanent innovations, (B) transitory innovations. Posterior mean and $67 \%$ band are plotted over time.

similar pattern of the low-high-low volatility shifts for GDP deflator inflation. The high volatility of the transitory innovations appears to contribute to the large fluctuations since the early 2000 s because the volatility of the permanent innovations is moderate during this period. This empirical finding is consistent with the result based on the multi-group changepoint model in the sense that CPI inflation experienced a dramatic drop in persistence around the early 1980s and the permanent innovations would not play an important role in generating high levels of CPI inflation volatility since the early 2000s.

\section{Summary and comparison with the literature}

To summarize, two different measures of inflation undergo distinct structural changes in different parameters as well as at different dates. Both measures of inflation switched from a low volatility regime to a high volatility regime in the early 1970s but switched to another low volatility regime at different dates: the early 1980s for GDP deflator inflation and the early 1990s for CPI inflation. Moreover, the residual variance for CPI inflation has increased again since the early 2000s but GDP deflator inflation has remained less volatile. The empirical results on the changes in volatility for two measures of inflation are robust to the alternative specification of stochastic volatility. CPI inflation experienced a dramatic drop in persistence around the early 
1980s but GDP deflator inflation is still persistent. The correlations between CPI inflation and GDP deflator inflation are calculated for the periods before and after 1981:Q3, which is the break date (posterior mode) for persistence of CPI inflation. The correlation has dropped substantially from 0.88 to 0.44 and this decrease also supports the different dynamics of the two inflation measures since the early 1980s. These findings about the complex patterns of structural changes reflect the possibility that the parameter shifts are caused by a variety of sources.

When reviewing recent findings in the literature, ${ }^{17}$ there appears to be controversy surrounding changes in inflation persistence. The mixed statistical evidence on inflation persistence in this paper seems to be along the lines of the disagreement in the literature. However, there seems to be an interesting pattern of results in reading recent studies in the literature regardless of persistence measures and econometric models. That is, whether there was a change in persistence around the early 1980s depends on the measure of inflation used in the analysis.

For example, Pivetta and Reis (2007) use GDP deflator inflation and conclude that inflation persistence has been roughly constant and high over the past 40 years, which is the same as the empirical finding in this paper. ${ }^{18}$ Pivetta and Reis (2007) conduct extensive research and robustness checks to support their findings. They first consider a Bayesian time-varying parameter model which allows for time-varying intercept and persistence parameters. They then calculate median unbiased estimates using local-to-unity asymptotics for different measures of persistence. Based on the various empirical models and estimates, they conclude that there has been no evidence of a change in persistence since 1965. For CPI inflation, Kim, Nelson and Piger (2004) find a persistence fall in 1979:Q2 and Leybourne et al. (2003) detected a change in inflation dynamics from I(1) to I(0) in 1982:Q2.

While most studies focus on only a particular measure of inflation, some papers examine multiple measures of inflation. ${ }^{19}$ Stock (2002) finds no evidence of a structural break for GDP deflator inflation but detects a single structural break in the regression coefficients for CPI inflation around 1981 using the Quandt likelihood ratio test at a 10\% level. This break date is consistent with the estimated (posterior mode) break date based on the multiple-group changepoint approach. Benati (2008) and Fuhrer (2010) consider a broad range of inflation measures for various subsamples, which are determined by monetary regimes and the so-called "Great Moderation" break, respectively. Benati (2008) documents that there was a fall in persistence for CPI inflation during the post-Volcker stabilization period since 1982:Q4, while other measures of inflation including GDP deflator inflation continued to be persistent. Finally, Fuhrer (2010) finds that since the early 1980s CPI inflation has very different dynamics from other inflation measures such as the GNP deflator, the GDP deflator, the PCE deflator, the core CPI, and the core PCE deflator. He confirms this difference by considering "grid-bootstrap" median-unbiased estimates of persistence, results for structural break test in persistence, first-order autocorrelation, and LAR estimates.

17 Recently, Cogley, Primiceri and Sargent (2010) and Kang, Kim and Morley (2009) study the persistence of inflation gap defined by the difference between inflation and its stochastic trend which evolves as a driftless random walk and find that there are changes in inflation gap persistence. Note that this model specification implies that inflation is assumed to have a unit root. Thus, their focus is different from that in this paper and other studies in the literature discussed here. Also, see the discussion in Benati (2008).

18 One exception in the recent literature is Taylor's (2000) study which calculates the median-unbiased estimate for the largest autoregressive root (LAR). He finds that the LAR estimate for the period of 1960:Q2-1979:Q4 (0.94) was much higher than for the period of 1982:Q1 to 1999:Q4 (0.74). However, However, Pivetta and Reis (2007) argue that Taylor's (2000) finding could be driven by the anomaly of having the sample period of 1982-1983, in which there was the minor exception of a possible short-lived change, because their 14-year window LAR estimate in 1999:Q4 is very close to the estimate for the period of 1960:Q2-1979:Q4.

19 Levin and Piger (2006) estimate autoregressive models with structural breaks for various measures of inflation: GDP deflator, CPI, CPI core, and PCE using the sample period of 1984:Q3-2005:Q2. Note that their sample period starts after the persistence break date (1981:Q3) for CPI inflation found in this paper. They cannot reject the null of structural break in the intercept for individual measures, but they are able to reject the null of no shift in the intercept for a multivariate model considering all the measures of inflation with the estimated break date in 1991:Q1 by using a seemingly unrelated regression model. 


\section{Conclusion}

In this paper, I employ a Bayesian approach to making inferences about complicated patterns of structural breaks in inflation dynamics. I modify Chib's (1998) approach by allowing multiple parameters to undergo mutually independent structural breaks at different dates with the different number of breaks. Structural breaks are modeled as abrupt changes to identify potential regime shifts in economic structure such as a long-run inflation target, monetary policy, and price-setting behavior. I examine postwar quarterly US inflation rates based on the CPI and the GDP deflator. Both inflation measures show multiple structural breaks in the residual variance but they show quite distinctive patterns in terms of timing and the number of breaks, which are confirmed by the empirical results based on the UC-SV model.

Another interesting finding is that CPI inflation experienced a dramatic drop in persistence around the early 1980s, but GDP deflator inflation is still persistent. However, I do not find evidence of a structural shift in the unconditional mean for both measures of inflation. The marginal probability calculations for the number of breaks in each individual parameter group also strongly support the model selection results based on the Bayes factors.

When reviewing the recent literature, considerable controversy exists over the structural break in inflation persistence around the early 1980s but the existence of the structural break appears to be dependent on the measures of inflation, as highlighted by the empirical findings in this paper. The structural breaks at different dates reflect the possibility that the parameter shifts are caused by a variety of factors.

Acknowledgments: I am very grateful to James Morley for his constructive discussion and comments. I also thank the editor and two anonymous referees, Kyu Ho Kang, Jeremy Piger, Anastasia Zervou, and participants at the Midwest Econometrics Group meeting and the Annual Symposium of the Society for Nonlinear Dynamics and Econometrics for helpful comments and suggestions. I acknowledge the use of GAUSS code written by James Stock and Mark Watson for unobserved components model with stochastic volatility. An earlier version of this paper was circulated under the title "Bayesian Inference about the Types of Structural Breaks When There Are Different Breaks in Many Parameters.”

\section{A. Appendix}

\section{A.1 Sampling posteriors}

I describe how to sample the latent regime indicators, $\left(\tilde{S}_{1, T}, \tilde{S}_{2, T}, \tilde{S}_{3, T}\right)$ and the posterior of model parameters conditional on the regime transition probabilities, $\boldsymbol{P}=\left(P_{1}, P_{2}, P_{3}\right)$ in the case of three parameter groups based on MCMC sampling algorithm in Section 2.

\section{A.1.1 Simulation of latent regime indicator $\tilde{S}_{g, T}$ for $g=1,2,3$}

The discrete latent regime indicators $\left\{S_{g, t}\right\}$ for $t=1, \ldots, T$ and $g=1,2,3$ are simulated in each step. The objective is to sample the indicators from the mass discrete function $p\left(\tilde{S}_{g, T} \mid \boldsymbol{\theta}, \tilde{S}_{-g, T}, \boldsymbol{P}, Y_{T}\right)$ where $p(\cdot)$ denotes a discrete mass function and $\tilde{S}_{-g, T}=\left(\tilde{S}_{1, T}, \ldots, \tilde{S}_{g-1, T}, \tilde{S}_{g+1, T}, \ldots, \tilde{S}_{G, T}\right)$. The mass function can be expressed as a joint density in reverse time order as follows.

$$
\begin{aligned}
p\left(\tilde{S}_{g, T} \mid \boldsymbol{\theta}, \tilde{S}_{-g, T}, \boldsymbol{P}, Y_{T}\right)= & p\left(S_{g, T} \mid \boldsymbol{\theta}, \tilde{S}_{-g, T}, \boldsymbol{P}, Y_{T}\right) \times p\left(S_{g, T-1} \mid S_{g, T}, \boldsymbol{\theta}, \tilde{S}_{-g, T}, \boldsymbol{P}, Y_{T}\right) \times \cdots \\
& \times p\left(S_{g, t} \mid S_{g}^{t+1}, \boldsymbol{\theta}, \tilde{S}_{-g, T}, \boldsymbol{P}, Y_{T}\right) \times \cdots \times p\left(S_{g, 1} \mid S_{g}^{2}, \boldsymbol{\theta}, \tilde{S}_{-g, T}, \boldsymbol{P}, Y_{T}\right)
\end{aligned}
$$

where $S_{g}^{t+1}=\left[S_{g, t+1} \ldots S_{g, T}\right]^{\prime}$. Notice that the first regime and the last regime are always one and $M_{g}+1$, respectively. These imply that for $g=1,2,3$ 


$$
p\left(S_{g, T}=M_{g}+1 \mid \theta, \tilde{S}_{-g, T}, P, Y_{T}\right)=1 \text { and } p\left(S_{g, 1}=1 \mid \theta, \tilde{S}_{-g, T}, P, Y_{T}\right)=1
$$

Thus, the regimes $S_{g, t}$ for $t=2, \ldots, t-1$ are recursively simulated from $t=T-1$ to $t=2$ in reverse time order.

As discussed in Section 2, the regime transition follows a first order Markov process. It is also independent of its own parameter as well as both other parameters and their latent regime indicators, as shown in Chib (1996). Thus, a term in (A.1) can be written that for $g=1,2,3$

$$
p\left(S_{g, t} \mid Y_{T}, S_{g}^{t+1}, \tilde{S}_{-g, T}, \boldsymbol{\theta}, \boldsymbol{P}\right) \propto p\left(S_{g, t} \mid Y_{t}, \tilde{S}_{-g, T}, \boldsymbol{\theta}, \boldsymbol{P}\right) \times p\left(S_{g, t+1} \mid S_{g, t}, P_{g}\right) .
$$

The first term in the proportion of the regime distribution is calculated recursively. Suppose $p\left(S_{g, t-1} \mid Y_{t-1}, \tilde{S}_{-g, T}, \boldsymbol{\theta}, \boldsymbol{P}\right)$ is known. Then, Bayes' rule can be applied for $k=1, \ldots, M_{g}+1$ regimes,

$$
p\left(S_{g, t}=k \mid Y_{t}, \tilde{S}_{-g, T}, \boldsymbol{\theta}, \boldsymbol{P}\right)=\frac{p\left(S_{g, t}=k \mid Y_{t-1}, \tilde{S}_{-g, T}, \boldsymbol{\theta}, \boldsymbol{P}\right) \times f\left(y_{t} \mid Y_{t-1}, \theta_{g, k}, \boldsymbol{\theta}_{-g}\right)}{\sum_{l=1}^{M_{g}+1} p\left(S_{g, t}=l \mid Y_{t-1}, \tilde{S}_{-g, T}, \boldsymbol{\theta}, \boldsymbol{P}\right) \times f\left(y_{t} \mid Y_{t-1}, \theta_{g, l}, \boldsymbol{\theta}_{-g}\right)}
$$

where

$$
p\left(S_{g, t}=k \mid Y_{t-1}, \tilde{S}_{-g, T}, \boldsymbol{\theta}, \boldsymbol{P}\right)=\sum_{l=k-1}^{k} p\left(S_{g, t}=k \mid S_{g, t-1}=l, P_{g}\right) \times p\left(S_{g, t-1}=l \mid Y_{t-1}, \tilde{S}_{-g, T}, \boldsymbol{\theta}, \boldsymbol{P}\right)
$$

and $p\left(S_{g, t}=k \mid S_{g, t-1}=l, P_{g}\right)$ is the $(l, k)$ th entry of the transition matrix $P_{g}$.

In sum, the probabilities of the regimes over dates are sampled through MCMC simulations:

$$
\operatorname{Pr}\left(S_{g, t}=k \mid Y_{T}\right)=\int p\left(S_{g, t}=k \mid Y_{T}, \boldsymbol{\theta}, \boldsymbol{P}\right) \pi\left(\boldsymbol{\theta}, \boldsymbol{P} \mid Y_{T}\right) d(\boldsymbol{\theta}, \boldsymbol{P})
$$

and in practice with $J$ simulations

$$
\operatorname{Pr}\left(S_{g, t}=k \mid Y_{T}\right)=\frac{1}{J} \sum_{j=1}^{J} p\left(S_{g, t}^{(j)}=k \mid Y_{T}, \boldsymbol{\theta}^{(j)}, \boldsymbol{P}^{(j)}\right) .
$$

\section{A.1.2 Simulation of transition probability matrix $\boldsymbol{P}_{\mathrm{g}}$}

The transition probability matrices $\left(P_{1}, P_{2}, P_{3}\right)$ are sampled only conditional on their regime indicators $\left(\tilde{S}_{1, T}, \tilde{S}_{2, T}, \tilde{S}_{3, T}\right)$, respectively. The reason is that the full conditional distribution $P_{g} \mid \theta, \tilde{S}_{g, T}, \tilde{S}_{-g, T}, P_{-g}, Y_{T}$ is independent of $\left(\theta, \tilde{S}_{-g, T}, P_{-g}, Y_{T}\right)$ where $P_{-g}=\left(P_{1}, \ldots, P_{g-1}, P_{g+1}, \ldots, P_{G}\right)$ for $g=1,2,3$. Thus, it can be shown that

$$
\pi\left(P_{g} \mid \theta, \tilde{S}_{g, T}, \tilde{S}_{-g, T}, P_{-g}, Y_{T}\right)=\pi\left(P_{g} \mid \tilde{S}_{g, T}\right) .
$$

If Beta priors for $p_{i, i}, i=1, \ldots, M_{g}$, are employed as

$$
p_{i, i} \sim \operatorname{Beta}\left(u_{i, i}, u_{i, i+1}\right)
$$

where $u_{i, i}$ and $u_{i, i+1}$ are the hyper-parameters, the posterior distribution can be derived as

$$
p_{i, i} \mid \tilde{S}_{g, T} \sim \operatorname{Beta}\left(u_{i, i}+n_{i, i}, u_{i, i+1}+n_{i, i+1}\right)
$$

where $n_{i, j}$ refers to the total number of transitions from regime $i$ to regime $j$. Note that $n_{i, i+1}$, for $i=1, \ldots, M_{g}$, is always equal to one since every regime never comes back to the previous regimes and moves to the next regime only once. For details, see Albert and Chib (1993).

\section{A.1.3 Sampling of the unconditional mean $\mu$}

Consider an AR(2) model with structural breaks in the unconditional mean, persistence coefficients, and the residual variance independently as follows: 


$$
\pi_{t}-\mu_{S_{1, t}}=\phi_{1, S_{2, t}}\left(\pi_{t-1}-\mu_{S_{1, t-1}}\right)+\phi_{2, S_{2, t-2}}\left(\pi_{t-2}-\mu_{S_{1,-2}}\right)+e_{t}, e_{t} \sim N\left(0, \sigma_{s_{3, t}}^{2}\right) .
$$

Conditional on $\left(\tilde{S}_{1, T}, \tilde{S}_{2, T}, \tilde{S}_{3, T}\right), \tilde{\phi}=\left(\phi_{1,1}, \phi_{2,1}, \ldots, \phi_{1, M_{2}+1}, \phi_{2, M_{2}+1}\right)$, and $\tilde{\sigma}^{2}=\left(\sigma_{1}^{2}, \ldots, \sigma_{M_{3}+1}^{2}\right)$, the unconditional mean for the regime $j, \mu_{j}$, for $j=1, \ldots,\left(M_{1}+1\right)$ can be sampled as follows.

(a) Prior

$$
\mu_{j} \sim N\left(\underline{\mu}_{j}, \underline{D}_{\mu_{j}}\right)
$$

(b) Posterior

$$
\mu_{j} \mid \tilde{\mu}_{-j}, \tilde{\phi}, \tilde{\sigma}^{2}, \tilde{S}_{1, T}, \tilde{S}_{2, T}, \tilde{S}_{3, T} \sim N\left(\bar{\mu}_{j}, \bar{D}_{\mu_{j}}\right)
$$

where $\tilde{\mu}_{-j}=\left(\mu_{1}, \ldots, \mu_{j-1}, \mu_{j+1}, \ldots, \mu_{M_{1}+1}\right)$,

$$
\begin{gathered}
\bar{\mu}_{j}=\left(\underline{D}_{\mu_{j}}^{-1}+\sum_{\left\{S_{1, t}=j, S_{1, t-1}=j, S_{1, t-2}=j\right\}} x_{1, t} x_{1, t}^{\prime} / \sigma_{S_{3, t}}^{2}\right)^{-1}\left(\underline{D}_{\mu_{j}}^{-1} \underline{\mu}_{j}+\sum_{\left\{S_{1, t}=j, S_{1, t-1}=j, S_{1, t-2}=j\right\}} x_{1, t} y_{1, t} / \sigma_{S_{3, t}}^{2}\right), \\
\bar{D}_{\mu_{j}}=\left(\underline{D}_{\mu_{j}}^{-1}+\sum_{\left\{S_{1, t}=j, S_{1, t-1}=j, S_{1, t-2}=j\right\}} x_{1, t} x_{1, t}^{\prime} / \sigma_{S_{3, t}^{2}}\right)^{-1}, \\
y_{1, t}=\pi_{t}-\phi_{1, S_{2, t}} \pi_{t-1}-\phi_{2, S_{2, t}} \pi_{t-2}-\mu_{S_{1, t}} 1\left(S_{1, t} \neq j\right)+\phi_{1, S_{2, t}} \mu_{S_{1, t-1}} 1\left(S_{1, t-1} \neq j\right)+\phi_{2, S_{2, t}} \mu_{S_{1, t-2}} 1\left(S_{1, t-2} \neq j\right),
\end{gathered}
$$

and

$$
x_{1, t}=1\left(S_{1, t}=j\right)-\phi_{1, S_{2, t}} 1\left(S_{1, t-1}=j\right)-\phi_{2, S_{2, t}} 1\left(S_{1, t-2}=j\right) .
$$

\section{A.1.4 Sampling of persistence coefficients $\phi$}

Conditional on $\left(\tilde{S}_{1, T}, \tilde{S}_{2, T}, \tilde{S}_{3, T}\right), \tilde{\mu}$, and $\tilde{\sigma}^{2}$, persistence parameters for regime $j, \phi_{j}=\left(\phi_{1, j}, \phi_{2, j}{ }^{\prime}\right.$, for $j=1, \ldots$, $\left(M_{2}+1\right)$ can be sampled as follows.

(a) Prior

$$
\phi_{j} \sim N\left(\underline{\phi}_{j}, \underline{D}_{\phi_{j}}\right)
$$

(b) Posterior

$$
\phi_{j} \mid \tilde{\mu}, \tilde{\sigma}^{2}, \tilde{S}_{1, T}, \tilde{S}_{2, T}, \tilde{S}_{3, T} \sim N\left(\bar{\phi}_{j}, \bar{D}_{\phi_{j}}\right)
$$

where

$$
\begin{gathered}
\bar{\phi}_{j}=\left(\underline{D}_{\phi_{j}}^{-1}+\sum_{\left\{S_{2, t}=j\right\}} x_{2, t} x_{2, t}^{\prime} / \sigma_{S_{3, t}^{2}}^{2}\right)^{-1}\left(\underline{D}_{\phi_{j}}^{-1} \underline{\phi}_{j}+\sum_{\left\{S_{2, t}=j\right\}} x_{2, t} y_{2, t} / \sigma_{S_{3, t}}^{2}\right), \\
\bar{D}_{\phi_{j}}=\left(\underline{D}_{\phi_{j}}^{-1}+\sum_{\left\{S_{2, t}=j\right\}} x_{2, t} x_{2, t}^{\prime} / \sigma_{S_{3, t}^{2}}^{2}\right)^{-1}, \\
y_{2, t}=\pi_{t}-\mu_{S_{1, t}},
\end{gathered}
$$

and

$$
x_{2, t}=\left[\left(\pi_{t-1}-\mu_{s_{1, t-1}}\right)\left(\pi_{t-2}-\mu_{s_{1,-2}}\right)\right]^{\prime}
$$

\section{A.1.5 Sampling of the residual variance $\sigma^{2}$}

Conditional on $\left(\tilde{S}_{1, T}, \tilde{S}_{2, T}, \tilde{S}_{3, T}\right), \tilde{\mu}$, and $\tilde{\phi}$, residual variance for regime $j, \sigma_{j}^{2}$, for $j=1, \ldots,\left(M_{3}+1\right)$ can be sampled as follows. 
(a) Prior

$$
\sigma_{j}^{2} \sim I G\left(\frac{v_{j}}{2}, \frac{\delta_{j}}{2}\right)
$$

(b) Posterior

$$
\sigma_{j}^{2} \mid \tilde{\mu}, \tilde{\phi}, \tilde{S}_{1, T}, \tilde{S}_{2, T}, \tilde{S}_{3, T} \sim I G\left(\frac{v_{j}+n_{j}}{2}, \frac{\delta_{j}+\sum_{\left\{S_{3, t}=j\right\}} e_{t}^{2}}{2}\right)
$$

where $n_{j}=\sum_{t=1}^{T} 1\left(S_{3, t}=j\right)$.

\section{A.2 Marginal likelihood calculation}

Let $m\left(Y_{T} \mid \mathcal{M}\right)$ is the marginal likelihood or the marginal density of the data $Y_{T}$ under model $\mathcal{M}$. The marginal likelihood of model $\mathcal{M}$ can be easily calculated through the method of Chib (1995) for Gibbs sampling based on the Bayes rule identity:

$$
m\left(Y_{T} \mid \mathcal{M}\right)=\frac{f\left(Y_{T} \mid \psi, \mathcal{M}\right) \pi(\psi \mid \mathcal{M})}{\pi\left(\psi \mid Y_{T}, \mathcal{M}\right)}
$$

where $\boldsymbol{\psi}=(\boldsymbol{\theta}, \boldsymbol{P})$. The above identity holds for any point $\boldsymbol{\psi}$ in the parameter space since the left hand side is free of $\boldsymbol{\psi}$. Taking the logarithm of the marginal likelihood for computational convenience, the estimate of the marginal density at any particular point $\boldsymbol{\psi}^{*}$ is given by

$$
\begin{aligned}
& \ln \hat{m}\left(Y_{T} \mid \mathcal{M}\right) \\
& =\ln f\left(Y_{T} \mid \boldsymbol{\theta}^{*}, \boldsymbol{P}^{*}, \mathcal{M}\right)+\ln \pi\left(\boldsymbol{\theta}^{*}, \boldsymbol{P}^{*} \mid \mathcal{M}\right)-\ln \pi\left(\boldsymbol{\theta}^{*}, \boldsymbol{P}^{*} \mid Y_{T}, \mathcal{M}\right) .
\end{aligned}
$$

I explain all the terms in equation (A.3) in the following subsections. For simplicity, I drop the model indicator $\mathcal{M}$ from now on.

\section{A.2.1 Likelihood function}

The logarithm of likelihood function is given by

$$
\ln f\left(Y_{T} \mid \psi^{*}\right)=\sum_{t=1}^{T} \ln f\left(y_{t} \mid Y_{t-1}, \psi^{*}\right)
$$

where

$$
f\left(y_{t} \mid Y_{t-1}, \psi^{*}\right)=\sum_{S_{1, t=1}}^{M_{1}+1 M_{2}+1 \sum_{2, t=1}} \sum_{S_{3, t=1}}^{M_{3}+1} f\left(y_{t} \mid Y_{t-1}, S_{1, t}, S_{2, t}, S_{3, t}, \psi^{*}\right) \times p\left(S_{1, t}, S_{2, t}, S_{3, t} \mid Y_{t-1}, \psi^{*}\right)
$$

is the one-step ahead prediction density and $f\left(y_{t} \mid Y_{t-1}, S_{1, t}, S_{2, t}, S_{3, t}, \boldsymbol{\psi}^{*}\right)$ is the conditional density of $y_{t}$ given the composite of regimes $\left(S_{1, t}, S_{2, t}, S_{3, t}\right)$ as well as the posterior mean $\boldsymbol{\psi}^{*}$. Define a composite of regimes for all the parameters by $S_{t}=\left(S_{1, t}, S_{2, t}, S_{3, t}\right)$. Then, $p\left(S_{1, t}, S_{2, t}, S_{3, t} \mid Y_{t-1}, \psi^{*}\right)$ is the joint discrete mass function of the composite $S_{t}=\left(S_{1, t}, S_{2, t}, S_{3, t}\right)$ and the transition probability matrix for the composite of regimes $S_{t}$ is given by $P_{1} \otimes P_{2} \otimes P_{3}$ where $\otimes$ indicates the Kronecker product, $P$ is a $m$-by- $m$ square matrix, and the number of the composite of regimes $S_{t}$ is given by $m=\left(M_{1}+1\right) \times\left(M_{2}+1\right) \times\left(M_{3}+1\right)$. For more details, see Kim and Nelson (1999). 


\section{A.2.2 Prior density}

All the parameters are a priori assumed to be independent of one another and the logarithm of prior density is given by

$$
\ln \pi\left(\psi^{*} \mid M\right)=\ln \pi\left(\tilde{\mu}^{*}\right)+\ln \pi\left(\tilde{\phi}^{*}\right)+\ln \pi\left(\tilde{\sigma}^{2 *}\right)+\ln \pi\left(P_{1}^{*}\right)+\ln \pi\left(P_{2}^{*}\right)+\ln \pi\left(P_{3}^{*}\right) .
$$

\section{A.2.3 Posterior density}

In order to estimate the posterior ordinate $\pi\left(\boldsymbol{\theta}^{*}, \boldsymbol{P}^{*} \mid Y_{T}\right)$, I consider the conditional decomposition of the posterior density as in Chib (1998). Note that the latent variables $\left(\tilde{S}_{1, T}, \tilde{S}_{2, T}, \tilde{S}_{3, T}\right)$ are integrated out in the calculation of the posterior density in each step and throughout this reduced Gibbs run, $\tilde{\mu}^{*}, \tilde{\phi}^{*}, \tilde{\sigma}^{2 *}, P_{1}^{*}$, and $P_{2}^{*}$ are set equal to their posterior mean.

$$
\begin{aligned}
\pi\left(\boldsymbol{\theta}^{*}, \boldsymbol{P}^{*} \mid Y_{T}\right)= & \pi\left(\tilde{\mu}^{*} \mid Y_{T}\right) \times \pi\left(P_{1}^{*} \mid \tilde{\mu}^{*}, Y_{T}\right) \times \pi\left(\tilde{\phi}^{*} \mid \tilde{\mu}^{*}, P_{1}^{*}, Y_{T}\right) \times \pi\left(P_{2}^{*} \mid \tilde{\mu}^{*}, \tilde{\phi}^{*}, P_{1}^{*}, Y_{T}\right) \\
& \times \pi\left(\tilde{\sigma}^{2 *} \mid \tilde{\mu}^{*}, \tilde{\phi}^{*}, P_{1}^{*}, P_{2}^{*}, Y_{T}\right) \pi\left(P_{3}^{*} \mid \tilde{\mu}^{*}, \tilde{\phi}^{*}, \tilde{\sigma}^{2 *}, P_{1}^{*}, P_{2}^{*}, Y_{T}\right)
\end{aligned}
$$

where

$$
\begin{aligned}
& \pi\left(\tilde{\mu}^{*} \mid Y_{T}\right)=\int \pi\left(\tilde{\mu}^{*} \mid \tilde{\phi}, \tilde{\sigma}^{2}, P_{1}, P_{2}, P_{3}, \tilde{S}_{1, T}, \tilde{S}_{2, T}, \tilde{S}_{3, T}, Y_{T}\right) \\
& \quad \times \pi\left(\tilde{\phi}, \tilde{\sigma}^{2}, P_{1}, P_{2}, P_{3}, \tilde{S}_{1, T}, \tilde{S}_{2, T}, \tilde{S}_{3, T} \mid Y_{T}\right) d \tilde{\phi} d \tilde{\sigma}^{2} d P_{1} d P_{2} d P_{3} d \tilde{S}_{1, T} d \tilde{S}_{2, T} d \tilde{S}_{3, T}, \\
& \pi\left(P_{1}^{*} \mid \tilde{\mu}^{*}, Y_{T}\right)=\int \pi\left(P_{1}^{*} \mid \tilde{\mu}^{*}, \tilde{\phi}_{,}, \tilde{\sigma}^{2}, P_{2}, P_{3}, \tilde{S}_{1, T}, \tilde{S}_{2, T}, \tilde{S}_{3, T}, Y_{T}\right) \\
& \quad \times \pi\left(\tilde{\phi}, \tilde{\sigma}^{2}, P_{2}, P_{3}, \tilde{S}_{1, T}, \tilde{S}_{2, T}, \tilde{S}_{3, T} \mid \tilde{\mu}^{*}, Y_{T}\right) d \tilde{\phi} d \tilde{\sigma}^{2} d P_{2} d P_{3} d \tilde{S}_{1, T} d \tilde{S}_{2, T} d \tilde{S}_{3, T}, \\
& \pi\left(\tilde{\phi}^{*} \mid \tilde{\mu}^{*}, P_{1}^{*}, Y_{T}\right)=\int \pi\left(\tilde{\phi}^{*} \mid \tilde{\mu}^{*}, P_{1}^{*}, \tilde{\phi}, \tilde{\sigma}^{2}, P_{2}, P_{3}, \tilde{S}_{1, T}, \tilde{S}_{2, T}, \tilde{S}_{3, T}, Y_{T}\right) \\
& \quad \times \pi\left(\tilde{\sigma}^{2}, P_{2}, P_{3}, \tilde{S}_{1, T}, \tilde{S}_{2, T}, \tilde{S}_{3, T} \mid \tilde{\mu}^{*}, P_{1}^{*}, Y_{T}\right) d \tilde{\sigma}^{2} d P_{2} d P_{3} d \tilde{S}_{1, T} d \tilde{S}_{2, T} d \tilde{S}_{3, T}, \\
& \pi\left(P_{2}^{*} \mid \tilde{\mu}^{*}, \tilde{\phi}^{*}, P_{1}^{*}, Y_{T}\right)=\int \pi\left(P_{2}^{*} \mid \tilde{\mu}^{*}, \tilde{\phi}^{*}, P_{1}^{*}, \tilde{\sigma}^{2}, P_{3}, \tilde{S}_{1, T}, \tilde{S}_{2, T}, \tilde{S}_{3, T}, Y_{T}\right) \\
& \quad \times \pi\left(\tilde{\sigma}^{2}, P_{3}, \tilde{S}_{1, T}, \tilde{S}_{2, T}, \tilde{S}_{3, T} \mid \tilde{\mu}^{*}, \tilde{\phi}^{*}, P_{1}^{*}, Y_{T}\right) d \tilde{\sigma}^{2} d P_{3} d \tilde{S}_{1, T} d \tilde{S}_{2, T} d \tilde{S}_{3, T}, \\
& \pi\left(\tilde{\sigma}^{2 *} \mid \tilde{\mu}^{*}, \tilde{\phi}^{*}, P_{1}^{*}, P_{2}^{*}, Y_{T}\right)=\int \pi\left(\tilde{\sigma}^{2 *} \mid \tilde{\mu}^{*}, \tilde{\phi}^{*}, P_{1}^{*}, P_{2}^{*}, P_{2}, \tilde{S}_{1, T}, \tilde{S}_{2, T}, \tilde{S}_{3, T}, Y_{T}\right) \\
& \quad \times \pi\left(P_{3}, \tilde{S}_{1, T}, \tilde{S}_{2, T}, \tilde{S}_{3, T} \mid \tilde{\mu}^{*}, \tilde{\phi}^{*}, P_{1}^{*}, P_{2}^{*}, Y_{T}\right) d P_{3} d \tilde{S}_{1, T} d \tilde{S}_{2, T} d \tilde{S}_{3, T},
\end{aligned}
$$

and

$$
\begin{gathered}
\pi\left(P_{3}^{*} \mid \tilde{\mu}^{*}, \tilde{\phi}^{*}, \tilde{\sigma}^{2 *}, P_{1}^{*}, P_{2}^{*}, Y_{T}\right)=\int \pi\left(P_{3}^{*} \mid \tilde{\mu}^{*}, \tilde{\phi}^{*}, \tilde{\sigma}^{2 *}, P_{1}^{*}, P_{2}^{*}, \tilde{S}_{1, T}, \tilde{S}_{2, T}, \tilde{S}_{3, T}, Y_{T}\right) \\
\times \pi\left(\tilde{S}_{1, T}, \tilde{S}_{2, T}, \tilde{S}_{3, T} \mid \tilde{\mu}^{*}, \tilde{\phi}^{*}, \tilde{\sigma}^{2 *}, P_{1}^{*}, P_{2}^{*}, Y_{T}\right) d \tilde{S}_{1, T} d \tilde{S}_{2, T} d \tilde{S}_{3, T}
\end{gathered}
$$

The decomposition of the posterior density shows that the first ordinate $\pi\left(\tilde{\mu}^{*} \mid Y_{T}\right)$ can be calculated based on draws from the full Gibbs run, and $\pi\left(P_{1}^{*} \mid \tilde{\mu}^{*}, Y_{T}\right), \pi\left(\tilde{\phi}^{*} \mid \tilde{\mu}^{*}, P_{1}^{*}, Y_{T}\right)$, $\pi\left(P_{2}^{*} \mid \tilde{\mu}^{*}, \tilde{\phi}^{*}, P_{1}^{*}, Y_{T}\right), \pi\left(\tilde{\sigma}^{2 *} \mid \tilde{\mu}^{*}, \tilde{\phi}^{*}, P_{1}^{*}, P_{2}^{*}, Y_{T}\right)$, and $\pi\left(P_{3}^{*} \mid \tilde{\mu}^{*}, \tilde{\phi}^{*}, \tilde{\sigma}^{2 *}, P_{1}^{*}, P_{2}^{*}, Y_{T}\right)$ can be calculated from appropriate reduced Gibbs runs. The Monte Carlo estimate of each decomposition component based on draws from each Gibbs run is calculated as follows.

$$
\begin{gathered}
\pi\left(\tilde{\mu}^{*} \mid Y_{T}\right)=\frac{1}{J} \sum_{j=1}^{J} \pi\left(\tilde{\mu}^{*} \mid \tilde{\phi}^{(j)}, \tilde{\sigma}^{2(j)}, P_{1}^{(j)}, P_{2}^{(j)}, P_{3}^{(j)}, \tilde{S}_{1, T}^{(j)}, \tilde{S}_{2, T}^{(j)}, \tilde{S}_{3, T}^{(j)}, Y_{T}\right), \\
\pi\left(P_{1}^{*} \mid \tilde{\mu}^{*}, Y_{T}\right)=\frac{1}{J} \sum_{j_{1}=1}^{J} \pi\left(P_{1}^{*} \mid \tilde{\mu}^{*}, \tilde{\phi}^{\left(j_{1}\right)}, \tilde{\sigma}^{2\left(j_{1}\right)}, P_{2}^{\left(j_{1}\right)}, P_{3}^{\left(j_{1}\right)}, Y_{T}\right),
\end{gathered}
$$




$$
\begin{gathered}
\pi\left(\tilde{\phi}^{*} \mid \tilde{\mu}^{*}, P_{1}^{*}, Y_{T}\right)=\frac{1}{J} \sum_{j_{2}=1}^{J} \pi\left(\tilde{\phi}^{*} \mid \tilde{\mu}^{*}, P_{1}^{*}, \tilde{\phi}^{\left(j_{2}\right)}, \tilde{\sigma}^{2\left(j_{2}\right)}, \tilde{S}_{1, T}^{\left(j_{2}\right)}, \tilde{S}_{2, T}^{\left(j_{2}\right)}, \tilde{S}_{3, T}^{\left(j_{2}\right)}, Y_{T}\right), \\
\pi\left(P_{2}^{*} \mid \tilde{\mu}^{*}, \tilde{\phi}^{*}, P_{1}^{*}, Y_{T}\right)=\frac{1}{J} \sum_{j_{3}=1}^{J} \pi\left(P_{2}^{*} \mid \tilde{\mu}^{*}, \tilde{\phi}^{*}, P_{1}^{*}, \tilde{\sigma}^{2\left(j_{3}\right)}, P_{3}^{\left(j_{3}\right)}, \tilde{S}_{1, T}^{\left(j_{3}\right)}, \tilde{S}_{2, T}^{\left(j_{3}\right)}, \tilde{S}_{3, T}^{\left(j_{3}\right)}, Y_{T}\right), \\
\pi\left(\tilde{\sigma}^{2 *} \mid \tilde{\mu}^{*}, \tilde{\phi}^{*}, P_{1}^{*}, P_{2}^{*}, Y_{T}\right)=\frac{1}{J} \sum_{j_{4}=1}^{J} \pi\left(\tilde{\sigma}^{2 *} \mid \tilde{\mu}^{*}, \tilde{\phi}^{*}, P_{1}^{*}, P_{2}^{*}, P_{3}^{\left(j_{4}\right)}, \tilde{S}_{1, T}^{\left(j_{4}\right)}, \tilde{S}_{2, T}^{\left(j_{4}\right)}, \tilde{S}_{3, T}^{\left(j_{4}\right)}, Y_{T}\right),
\end{gathered}
$$

and

$$
\pi\left(P_{3}^{*} \mid \tilde{\mu}^{*}, \tilde{\phi}^{*}, \tilde{\sigma}^{2 *}, P_{1}^{*}, P_{2}^{*}, Y_{T}\right)=\frac{1}{J} \sum_{j_{5}=1}^{J} \pi\left(P_{3}^{*} \mid \tilde{\mu}^{*}, \tilde{\phi}^{*}, \tilde{\sigma}^{2 *}, P_{1}^{*}, P_{2}^{*}, \tilde{S}_{1, T}^{\left(j_{5}\right)}, \tilde{S}_{2, T}^{\left(j_{5}\right)}, \tilde{S}_{3, T}^{\left(j_{5}\right)}, Y_{T}\right)
$$

where the superscript $(j)$ refers to the $j$ th draw of the full Gibbs run and the superscript $(j), i=1, \ldots, 5$, refers to the $j_{i}$ th draw from the appropriate reduced Gibbs run. Thus, in addition to the full Gibbs run for the usual estimation of parameters, it is required to have five additional Gibbs runs ( $5 \times J$ iterations). For example, $\pi\left(\tilde{\sigma}^{2 *} \mid \tilde{\mu}^{*}, \tilde{\phi}^{*}, P_{1}^{*}, P_{2}^{*}, Y_{T}\right)$ is calculated by additional $J$ iterations from the following reduced $j_{4}$ th Gibbs run.

Algorithm for $\pi\left(\tilde{\sigma}^{2 *} \mid \tilde{\mu}^{*}, \tilde{\phi}^{*}, P_{1}^{*}, P_{2}^{*}, Y_{T}\right)$

(i) Generate $\tilde{\sigma}^{2\left(\mathrm{j}_{4}\right)}$ from $\pi\left(\tilde{\sigma}^{2} \mid \tilde{\mu}^{*}, \tilde{\phi}^{*}, P_{1}^{*}, P_{2}^{*}, P_{3}^{\left(j_{4}-1\right)}, S_{1, T}^{\left(j_{4}-1\right)}, S_{2, T}^{\left(j_{4}-1\right)}, S_{3, T}^{\left(j_{4}-1\right)}, Y_{T}\right)$

(ii) Generate $P_{3}^{\left(j_{4}\right)}$ from $\pi\left(P_{3} \mid \tilde{\mu}^{*}, \tilde{\phi}^{*}, P_{1}^{*}, P_{2}^{*}, \tilde{\sigma}^{2\left(j_{4}\right)}, S_{1, T}^{\left(j_{4}-1\right)}, S_{2, T}^{\left(j_{4}-1\right)}, S_{3, T}^{\left(j_{4}-1\right)}, Y_{T}\right)$

(iii) Generate $\tilde{S}_{1, T}^{\left(j_{4}\right)}$ from $\pi\left(S_{1, T} \mid \tilde{\mu}^{*}, \tilde{\phi}^{*}, P_{1}^{*}, P_{2}^{*}, \tilde{\sigma}^{2\left(j_{4}\right)}, P_{3}^{\left(j_{4}\right)}, S_{2, T}^{\left(j_{4}-1\right)}, S_{3, T}^{\left(j_{4}-1\right)}, Y_{T}\right)$

(iv) Generate $\tilde{S}_{2, T}^{\left(j_{4}\right)}$ from $\pi\left(S_{2, T} \mid \tilde{\mu}^{*}, \tilde{\phi}^{*}, P_{1}^{*}, P_{2}^{*}, \tilde{\sigma}^{2\left(j_{4}\right)}, P_{3}^{\left(j_{4}\right)}, S_{1, T}^{\left(j_{4}\right)}, S_{3, T}^{\left(j_{4}-1\right)}, Y_{T}\right)$

(v) Generate $\tilde{S}_{3, T}^{\left(j_{4}\right)}$ from $\pi\left(S_{3, T} \mid \tilde{\mu}^{*}, \tilde{\phi}^{*}, P_{1}^{*}, P_{2}^{*}, \tilde{\sigma}^{2\left(j_{4}\right)}, P_{3}^{\left(j_{4}\right)}, S_{1, T}^{\left(j_{4}\right)}, S_{2, T}^{\left(j_{4}\right)}, Y_{T}\right)$

(vi) Evaluate $\pi\left(\tilde{\sigma}^{2 *} \mid \tilde{\mu}^{*}, \tilde{\phi}^{*}, P_{1}^{*}, P_{2}^{*}, P_{3}^{\left(j_{4}\right)}, S_{1, T}^{\left(j_{4}\right)}, S_{2, T}^{\left(j_{4}\right)}, \tilde{S}_{3, T}^{\left(j_{4}\right)}, Y_{T}\right)$

\section{References}

Albert, James H., and Siddhartha Chib. 1993. "Bayes inference via Gibbs Sampling of Autoregressive Time Series Subject to Markov Mean and Variance Shifts." Journal of Business and Economic Statistics 11: 1-15.

Bai, Jushan, and Pierre Perron. 2003. "Computation and Analysis of Multiple Structural Change Models." Journal of Applied Econometrics 18 (1): 1-22.

Bauwens, Luc, Gary Koop, Dimitris Korobilis, and Jeroen V. K. Rombouts. 2015. "The Contribution of Structural Break Models to Forecasting Macroeconomic Series.” Journal of Applied Econometrics 30 (4): 596-620.

Benati, Luca. 2008. “Investigating Inflation Persistence across Monetary Regimes.” The Quarterly Journal of Economics 123 (3): $1005-1060$.

Chib, Siddhartha. 1995. "Marginal Likelihood from the Gibbs Output.” Journal of the American Statistical Association 90: $1313-1321$.

Chib, Siddhartha. 1996. "Calculating Posterior Distributions and Modal Estimates in Markov Mixture Models." Journal of Econometrics 75 (1): 79-97.

Chib, Siddhartha. 1998. "Estimation and Comparison of Multiple Change-point Models." Journal of Econometrics 86 (2): 221-241.

Cogley, Timothy, and Thomas J. Sargent. 2002. "Evolving Post-world War II US Inflation Dynamics." In NBER Macroeconomics Annual 2001, Volume 16. 331-388. Cambridge, MA: MIT Press.

Cogley, Timothy, Giorgio E. Primiceri, and Thomas J. Sargent. 2010. “Inflation-Gap Persistence in the US.” American Economic Journal: Macroeconomics 2 (1): 43-69.

Davig, Troy, and Taeyoung Doh. 2014. "Monetary Policy Regime Shifts and Inflation Persistence." Review of Economics and Statistics 96 (5): 862-875. 
Fuhrer, Jeff, and George Moore. 1995. “Inflation Persistence.” The Quarterly Journal of Economics 110 (1): 127-159.

Fuhrer, Jeffrey C. 2010. “Inflation Persistence.” Handbook of Monetary Economics 3: 423-486.

Giordani, Paolo, and Robert Kohn. 2008. "Efficient Bayesian Inference for Multiple Change-point and Mixture Innovation Models."Journal of Business \& Economic Statistics 26 (1): 66-77.

Jeffreys, Harold. 1961. The Theory of Probability. London; New York; Oxford, UK: Oxford University Press.

Kang, Kyu Ho, Chang-Jin Kim, and James Morley. 2009. “Changes in US Inflation Persistence.” Studies in Nonlinear Dynamics \& Econometrics 13(4).

Kim, Chang-Jin, and Charles R. Nelson. 1999. State-Space Models with Regime Switching: Classical and Gibbs-Sampling Approaches with Applications. Cambridge, MA: The MIT Press.

Kim, Chang-Jin, Charles R. Nelson, and Jeremy Piger. 2004. "The Less-volatile US Economy: A Bayesian Investigation of Timing, Breadth, and Potential Explanations." Journal of Business \& Economic Statistics 22 (1): 80-93.

Koop, Gary, and Simon M. Potter. 2007. "Estimation and Forecasting in Models with Multiple Breaks." The Review of Economic Studies 74 (3): 763-789.

Levin, Andrew, and Jeremy Piger. 2006. “Is Inflation Persistence Intrinsic in Industrial Economies?”.

Levin, Andrew, and Jeremy Piger. 2007. "Bayesian Model Selection for Structural Break Models.” Manuscript.

Leybourne, Stephen, Tae-Hwan Kim, Vanessa Smith, and Paul Newbold. 2003. "Tests for a change in persistence against the null of difference-stationarity.” The Econometrics Journal 6 (2): 291-311.

Pesaran, M. Hashem, Davide Pettenuzzo, and Allan Timmermann. 2006. "Forecasting Time Series Subject to Multiple Structural Breaks." The Review of Economic Studies 73 (4): 1057-1084.

Pivetta, Frederic, and Ricardo Reis. 2007. "The Persistence of Inflation in the United States." Journal of Economic Dynamics and Control 31: 1326-1358.

Sims, Christopher A. 2001. “Comment on Sargent and Cogley’s ‘Evolving Post World War II US Inflation Dynamics'.” NBER Macroeconomics Annual 16: 373-379.

Sims, Christopher A., and Tao Zha. 2006. "Were There Regime Switches in US Monetary Policy?" The American Economic Review 96 (1): 54-81.

Smets, Frank, and Raf Wouters. 2007. "Shocks and Frictions in US Business Cycles: A Bayesian DSGE Approach." American Economic Review 97 (3): 586-606.

Solow, Robert M. 1968. “Recent Controversy on the Theory of Inflation: An Eclectic View." Inflation: Its Causes, Consequences, and Control, edited by Stephen W. Rousseas. New York: New York University.

Stock, James. 2001. “Comment on Sargent and Cogley's ‘Evolving post World War II US Inflation Dynamics'.” NBER Macroeconomics Annual 16: 379-387.

Stock, James. 2002. “Comment on Evolving Post-World War II U.S. Inflation Dynamics.” NBER Macroeconomics Annual 2001 379-387.

Stock, James H., and Mark W. Watson. 2003. “Has the Business Cycle Changed, and Why?” NBER Macroeconomics Annual 2002 17: $159-218$.

Stock, James H., and Mark W. Watson. 2007. "Why has US Inflation Become Harder to Forecast?” Journal of Money, Credit and banking 39 (s1): 3-33.

Taylor, John B. 2000. “Low Inflation, Pass-through, and the Pricing Power of Firms.” European Economic Review 44 (7): 1389-1408.

Tobin, James. 1968. “Discussion.” In Proceedings of a Symposium on Inflation: Its Causes, Consequences, and Control, edited by S. Rousseaus. New York: New York University.

Wang, Jiahui, and Eric Zivot. 2000. “A Time Series Model of Multiple Structural Changes in Level, Trend and Variance.” Journal of Business and Economic Statistics 18 (3): 374-386.

Supplemental Material: The online version of this article (DOI: 10.1515/snde-2015-0041) offers supplementary material, available to authorized users. 